\title{
A Reevaluation of the Mechanism of the Amination of Aryl Halides Catalyzed by BINAP-Ligated Palladium Complexes
}

\author{
Shashank Shekhar, ${ }^{\ddagger}$ Per Ryberg, ${ }^{\ddagger}$ John F. Hartwig ${ }^{\ddagger \star}$ \\ ${ }^{*}$ Department of Chemistry, Yale University, P.O. Box 208107, New Haven, CT 06520-8107 \\ Jinu S. Mathew, ${ }^{\S}$ Donna G. Blackmond, ${ }^{\S *}$ \\ ${ }^{8}$ Department of Chemistry, Imperial College, London SW7 2AZ United Kingdom \\ Eric R. Strieter, ${ }^{\dagger}$ Stephen L. Buchwald ${ }^{\dagger \star}$ \\ 'Department of Chemistry, Massachusetts Institute of Technology, Cambridge, MA 02139
}

\section{Supplementary Material}

\section{Index}

Experimental Section 1. Experiments carried out by the authors at Yale University. S2-S7

Experimental Section 2. Experiments carried out by the authors at Massachusetts Institute of Technology and Imperial College, London.

Crystallographic data for $[(\text { BINAP }) P d]_{2}($ dba $)$.

S9-S35

Figure S1. Sequential reactions of 3-bromoanisole with $N$-methylpiperazine and $\mathrm{NaOtAm}$ as base with $\mathrm{Pd}(\mathrm{BINAP})_{2}$ and BINAP as catalyst at $70^{\circ} \mathrm{C}$.

Figure S2. Sequential reactions of bromobenzene with hexylamine and NaOtAm as base with $\mathrm{Pd}_{2}(\mathrm{dba})_{3}$ and BINAP as catalyst at $60^{\circ} \mathrm{C}$.

Figure S3. Sequential reactions of bromobenzene with $N$-methylpiperazine and NaOtAm as base with $\mathrm{Pd}_{2}(\mathrm{dba})_{3}$ and BINAP as catalyst at $60^{\circ} \mathrm{C}$.

Derivation of rate equations. 


\section{Experimental Section 1}

General Considerations. All manipulations were conducted in an inert atmosphere glove box unless otherwise noted. ${ }^{31} \mathrm{P}\left\{{ }^{1} \mathrm{H}\right\}$ NMR spectra were recorded at 202 or $161 \mathrm{MHz}$. Chemical shifts are reported in units of parts per million relative to $85 \% \mathrm{H}_{3} \mathrm{PO}_{4}$ as external standard. ${ }^{1} \mathrm{H}$ NMR spectra were recorded on a 400 NMR spectrometer, and chemical shifts are referenced to tetramethylsilane or residual protiated solvent. The temperature in the probe cavity was measured with a copper thermocouple inserted into toluene in an NMR sample tube in the probe. Unless specified otherwise, all reagents were purchased from commercial suppliers and used without further purification. $\operatorname{Pd}(\mathrm{BINAP})_{2},{ }^{7} \mathrm{Pd}\left[\mathrm{P}(o-\mathrm{Tol})_{3}\right]_{2}{ }^{30,31}$ and $(\mathrm{BINAP}) \mathrm{Pd}(\mathrm{dba})^{4}$ were prepared by literature procedures. Protiated solvents were heated to reflux and distilled from sodium benzophenone or were obtained as A.C.S. reagent grade and were degassed by purging with nitrogen for $30 \mathrm{~min}$ and dried by passing through a $1 \mathrm{~m}$ column containing activated alumina in a solvent purification system. Deuterated solvents were dried over sodiumbenzophenone ketyl and were collected by vacuum transfer. Octylamine, and $N$ methylpiperazine were distilled from $\mathrm{CaH}_{2}$.

Preparation of $[(\mathbf{B I N A P}) \mathbf{P d}]_{2}(\mathbf{d b a}) . \quad(\mathrm{BINAP}) \mathrm{Pd}(\mathrm{dba})(80 \mathrm{mg}, 0.083 \mathrm{mmol}), \mathrm{NaO} t \mathrm{Am}$ (21 mg, $0.19 \mathrm{mmol})$ and octylamine $(116 \mu \mathrm{L}, 0.669 \mathrm{mmol})$ were dissolved in $1 \mathrm{ml}$ of benzene and heated at $50^{\circ} \mathrm{C}$ for $1 \mathrm{~h}$. After the mixture had cooled to room temperature, $4 \mathrm{ml}$ of pentane was added. The precipitate that formed over $4 \mathrm{~h}$ was collected by filtration and dried in vacuum. The filtrate was evaporated to dryness, and the solid obtained was dissolved in $0.5 \mathrm{ml}$ of benzene and $4 \mathrm{ml}$ of pentane was added. The precipitate formed over $6 \mathrm{~h}$ was collected by filtration and dried in vacuum. The combined yield of product was $122 \mathrm{mg}(87 \%)$. Slow diffusion of pentane into a toluene solution of the product generated crystals suitable for X-ray diffraction. ${ }^{1} \mathrm{H}$ NMR 
(400 MHz, $\left.\mathrm{C}_{6} \mathrm{D}_{6}\right): \delta$ 7.89-7.82 (m), 7.72-7.69 (m), 7.59-7.43 (m), 7.39-7.35 (m), 7.31-7.08 (m), 7.08-6.88 (m), 6.86-6.81 (m), 6.79-6.70 (m), 6.26-6.55 (m), $6.32(\mathrm{~b})$, 5.06-5.01 (m), 4.92-4.87 (m). ${ }^{31} \mathrm{P}\left\{{ }^{1} \mathrm{H}\right\} \mathrm{NMR}\left(202 \mathrm{MHz}, \mathrm{C}_{6} \mathrm{D}_{6}\right.$ ): $\delta 28.1$ (app t, $9 \mathrm{~Hz}$ ), 26.4 (app t, $8 \mathrm{~Hz}$ ) Anal. Calcd. For $\mathrm{C}_{105} \mathrm{H}_{78} \mathrm{OP}_{4} \mathrm{Pd}_{2}: \mathrm{C} 74.51, \mathrm{H}$ 4.65. Found C 74.76, H 4.64.

Determination of resting state of the catalyst in reactions catalysed by $\operatorname{Pd}(\mathrm{BINAP})_{2}$. $\mathrm{Pd}(\mathrm{BINAP})_{2}\left(10 \mathrm{mg}, 7.4 \times 10^{-6} \mathrm{~mol}\right)$, octylamine $(75 \mu \mathrm{L}, 0.45 \mathrm{mmol})$ or $\mathrm{N}$-methylpiperazine $(50$ $\mu \mathrm{L}, 0.45 \mathrm{mmol}), \mathrm{BINAP}\left(2.0 \mathrm{mg}, 3.2 \times 10^{-6} \mathrm{~mol}\right)$, and $\mathrm{NaO} t \mathrm{Am}(55 \mathrm{mg}, 0.50 \mathrm{mmol})$ were weighed into a NMR tube with a screw cap lined with Teflon and $\mathrm{C}_{6} \mathrm{D}_{6}$ was added to give a total volume of $0.55 \mathrm{ml}$. A flame-sealed capillary tube containing $\mathrm{H}_{3} \mathrm{PO}_{4}$ in 1 ,4-dioxane $(20 \mathrm{mg} / \mathrm{mL})$ was inserted into the NMR tube. The NMR tube was placed into an NMR spectrometer probe that had been preheated at $60{ }^{\circ} \mathrm{C} . \mathrm{A}{ }^{31} \mathrm{P}\left\{{ }^{1} \mathrm{H}\right\}$ NMR spectrum was recorded. The NMR tube was removed from the probe and 3-bromoanisole $(50 \mu \mathrm{L}, 0.39 \mathrm{mmol})$ was added. The NMR tube was shaken and returned to the probe. ${ }^{31} \mathrm{P}\left\{{ }^{1} \mathrm{H}\right\}$ NMR spectra were recorded with 20 minute intervals until the reaction had occurred to more than $95 \%$ conversion.

Determination of resting state of the palladium in reactions catalysed by $[(\mathbf{B I N A P}) \mathbf{P d}]_{2}(\mathbf{d b a})$. The above procedure was followed using $[(\mathrm{BINAP}) \mathrm{Pd}]_{2}(\mathrm{dba})(12.7 \mathrm{mg}$, $\left.7.4 \times 10^{-6} \mathrm{~mol}\right)$, octylamine $(75 \mu \mathrm{L}, 0.45 \mathrm{mmol})$ or $\mathrm{N}$-methylpiperazine $(50 \mu \mathrm{L}, 0.45 \mathrm{mmol})$, and $\mathrm{NaOtAm}(60 \mathrm{mg}, 0.55 \mathrm{mmol})$.

Determination of resting state of the palladium in reactions catalysed by (BINAP)Pd(dba). (BINAP)Pd(dba) $\left(7 \mathrm{mg}, 7.4 \times 10^{-6} \mathrm{~mol}\right)$, octylamine $(75 \mu \mathrm{L}, 0.45 \mathrm{mmol})$ or $\mathrm{N}$-methylpiperazine $(50 \mu \mathrm{L}, 0.45 \mathrm{mmol}), \mathrm{NaOtAm}(60 \mathrm{mg}, 0.55 \mathrm{mmol})$ and 3-bromoanisole (50 $\mu \mathrm{L}, 0.39 \mathrm{mmol}$ ) were weighed into a NMR tube with a screw cap lined with Teflon and $\mathrm{C}_{6} \mathrm{D}_{6}$ was added to give a total volume of $0.55 \mathrm{ml}$. The NMR tube was vigorously shaken and a flame- 
sealed capillary tube containing $\mathrm{H}_{3} \mathrm{PO}_{4}$ in 1,4-dioxane $(20 \mathrm{mg} / \mathrm{mL})$ was inserted into the NMR tube. The NMR tube was placed into an NMR spectrometer probe that had been preheated at $60^{\circ}$ C. ${ }^{31} \mathrm{P}\left\{{ }^{1} \mathrm{H}\right\}$ NMR spectrum was recorded with 20 minute intervals until the reaction had occurred to more than $95 \%$ conversion.

Determination of resting state of the palladium in reactions catalysed by $\operatorname{Pd}_{2}(\mathrm{dba})_{3}$ and BINAP. The above procedure was followed using $\mathrm{Pd}_{2}(\mathrm{dba})_{3}\left(3.4 \mathrm{mg}, 3.7 \times 10^{-6} \mathrm{~mol}\right), R$ BINAP $\left(4.6 \mathrm{mg}, 7.4 \times 10^{-6} \mathrm{~mol}\right)$, octylamine $(75 \mu \mathrm{L}, 0.45 \mathrm{mmol})$ or N-methylpiperazine $(50 \mu \mathrm{L}$, $0.45 \mathrm{mmol})$, and $\mathrm{NaOtAm}(60 \mathrm{mg}, 0.55 \mathrm{mmol})$.

General procedure for sequential reactions of 3-bromoanisole with $N$ methylpiperazine and $\mathrm{NaOtAm}$ catalyzed by palladium complexes. $\mathrm{NaO} t \mathrm{Am}(75 \mathrm{mg}, 0.68$ mmol), palladium complex $\left(7.1 \times 10^{-6} \mathrm{~mol}\right)$ and 1,3,5-trioxane $(3.5 \mathrm{mg}, 0.040 \mathrm{mmol})$ (internal standard) were dissolved in $0.60 \mathrm{~mL} \mathrm{C}_{6} \mathrm{D}_{6}$ in an NMR tube with a screw cap lined with Teflon. $N$-Methylpiperazine $(75 \mu \mathrm{L}, 0.68 \mathrm{mmol})$ and 3-bromoanisole $(10 \mu \mathrm{L}, 0.079 \mathrm{mmol})$ were added by syringe into the NMR tube just before inserting the tube into a preheated spectrometer probe. The second, third and fourth portions of 3-bromoanisole (10 $\mu \mathrm{L}, 0.079 \mathrm{mmol})$ were added by syringe into the NMR tube when the resonances of the aryl bromide were no longer detected in the ${ }^{1} \mathrm{H}$ NMR spectrum. First-order rate constants were obtained by fitting a plot of the decay of the resonance of 3-bromoanisole versus time to the expression $\mathrm{A}=B e^{k t}+C$ in which $k$ is the first-order rate constant $k_{\mathrm{obs}}$

Sequential reactions of 3-bromoanisole with $N$-methylpiperazine and NaOtAm catalyzed by $[\mathbf{P d}(\mathbf{B I N A P})]_{2}(\mathbf{d b a})$. The above general procedure was followed with $[\mathrm{Pd}(\mathrm{BINAP})]_{2}(\mathrm{dba})\left(12 \mathrm{mg}, 7.1 \times 10^{-6} \mathrm{~mol}\right)$ as precatalyst at $50^{\circ} \mathrm{C}$. 
Sequential reactions of 3-bromoanisole with $\mathrm{N}$-methylpiperazine and $\mathrm{NaOtAm}$ catalyzed by (BINAP)Pd(dba). The above general procedure was followed using (BINAP)Pd(dba) $\left(6.8 \mathrm{mg}, 7.1 \times 10^{-6} \mathrm{~mol}\right)$ as catalyst at $65^{\circ} \mathrm{C}$.

Sequential reactions of 3-bromoanisole with $\mathrm{N}$-methylpiperazine and $\mathrm{NaOtAm}$ catalyzed by $\mathbf{P d}_{2}(\mathbf{d b a})_{3}$ and BINAP. The above general procedure was followed using $\operatorname{Pd}_{2}(\mathrm{dba})_{3}$ (3.3 $\left.\mathrm{mg}, 3.7 \times 10^{-6} \mathrm{~mol}\right)$ and $R$-BINAP $\left(4.7 \mathrm{mg}, 7.4 \times 10^{-6} \mathrm{~mol}\right)$ as catalyst at $65^{\circ} \mathrm{C}$

Sequential reactions of 3-bromoanisole with $\mathrm{N}$-methylpiperazine and $\mathrm{NaOtAm}$ catalyzed by Pd(BINAP) $)_{2}$. The above general procedure was followed using $\operatorname{Pd}(\mathrm{BINAP})_{2}(9.5$ $\left.\mathrm{mg}, 7.1 \times 10^{-6} \mathrm{~mol}\right)$ as catalyst at $50^{\circ} \mathrm{C}$.

Sequential reactions of 3-bromoanisole with $\mathrm{N}$-methylpiperazine and $\mathrm{NaOtAm}$ catalyzed by $\operatorname{Pd}(\mathrm{BINAP})_{2}$ in the presence of added BINAP $(2 \mathrm{~mol} \%)$. The reaction mixture was prepared by adding $100 \mu \mathrm{L}$ of a stock solution of BINAP $(15 \mathrm{mM})$ to a mixture of $\mathrm{NaOtAm}$ (75 mg, $0.68 \mathrm{mmol}), 1,3,5$-trioxane $(3.5 \mathrm{mg}, 0.040 \mathrm{mmol})$ and Pd(BINAP) $\left(9.5 \mathrm{mg}, 7.1 \times 10^{-6}\right.$ mol) in $\mathrm{C}_{6} \mathrm{D}_{6}(0.50 \mathrm{~mL})$. The reaction was conducted in an NMR tube with a screw cap lined with Teflon at $70^{\circ} \mathrm{C}$.

General procedure for sequential reactions of 3-bromoanisole with octylamine and NaOt $\mathrm{Am}$ catalyzed by palladium complexes. $\mathrm{NaO} t \mathrm{Am}(75 \mathrm{mg}, 0.68 \mathrm{mmol})$, palladium complex $\left(7.1 \times 10^{-6} \mathrm{~mol}\right)$ and 1,3,5-trioxane $(3.5 \mathrm{mg}, 0.040 \mathrm{mmol})$ (internal standard) were dissolved in $0.60 \mathrm{~mL} \mathrm{C} \mathrm{C}_{6} \mathrm{D}_{6}$ in an NMR tube with a screw cap lined with Teflon. Octylamine (115 $\mu \mathrm{L}, 0.69 \mathrm{mmol})$ and 3-bromoanisole $(10 \mu \mathrm{L}, 0.079 \mathrm{mmol})$ were added by syringe into the NMR tube just before inserting the tube into a preheated spectrometer probe. The second, third and fourth portions of 3-bromoanisole $(10 \mu \mathrm{L}, 0.079 \mathrm{mmol})$ were added by syringe into the NMR tube when the resonances of the aryl bromide were no longer detected in the ${ }^{1} \mathrm{H}$ NMR 
spectrum. First-order rate constants were obtained by fitting a plot of the decay of the resonance of 3-bromoanisole versus time to the expression $\mathrm{A}=B e^{k t}+C$ in which $k$ is the first-order rate constant $k_{\text {obs }}$

Sequential reactions of 3-bromoanisole with $N$-methylpiperazine and $\mathrm{NaOtAm}$ catalyzed by $[\mathbf{P d}(\mathbf{B I N A P})]_{2}(\mathbf{d b a})$. The above general procedure was followed with $[\mathrm{Pd}(\mathrm{BINAP})]_{2}(\mathrm{dba})\left(12 \mathrm{mg}, 7.1 \times 10^{-6} \mathrm{~mol}\right)$ as precatalyst at $50^{\circ} \mathrm{C}$.

Sequential reactions of 3-bromoanisole with $N$-methylpiperazine and $\mathrm{NaO} t \mathrm{Am}$ catalyzed by (BINAP)Pd(dba). The above general procedure was followed using (BINAP)Pd(dba) $\left(6.8 \mathrm{mg}, 7.1 \times 10^{-6} \mathrm{~mol}\right)$ as catalyst at $65^{\circ} \mathrm{C}$.

Sequential reactions of 3-bromoanisole with $N$-methylpiperazine and $\mathrm{NaO} t \mathrm{Am}$ catalyzed by $\mathbf{P d}_{2}(\mathbf{d b a})_{3}$ and BINAP. The above general procedure was followed using $\operatorname{Pd}_{2}(\mathrm{dba})_{3}$ (3.3 $\left.\mathrm{mg}, 3.7 \times 10^{-6} \mathrm{~mol}\right)$ and $R$-BINAP $\left(4.7 \mathrm{mg}, 7.4 \times 10^{-6} \mathrm{~mol}\right)$ as catalyst at $65^{\circ} \mathrm{C}$

Sequential reactions of 3-bromoanisole with $N$-methylpiperazine and $\mathrm{NaOtAm}$ catalyzed by Pd(BINAP) $)_{2}$. The above general procedure was followed using $\operatorname{Pd}(\mathrm{BINAP})_{2}(9.5$ $\left.\mathrm{mg}, 7.1 \times 10^{-6} \mathrm{~mol}\right)$ as catalyst at $50^{\circ} \mathrm{C}$.

Sequential reactions of 3-bromoanisole with $N$-methylpiperazine and NaOtAm catalyzed by Pd(BINAP $)_{2}$ in the presence of added BINAP ( 2 mol \%). The reaction mixture was prepared by adding $100 \mu \mathrm{L}$ of a stock solution of BINAP $(15 \mathrm{mM})$ to a mixture of $\mathrm{NaO} t \mathrm{Am}$ (75 mg, $0.68 \mathrm{mmol}), 1,3,5$-trioxane $(3.5 \mathrm{mg}, 0.040 \mathrm{mmol})$ and $\mathrm{Pd}(\mathrm{BINAP})_{2}\left(9.5 \mathrm{mg}, 7.1 \times 10^{-6}\right.$ mol) in $\mathrm{C}_{6} \mathrm{D}_{6}(0.50 \mathrm{~mL})$. The reaction was conducted in an NMR tube with a screw cap lined with Teflon at $70^{\circ} \mathrm{C}$.

Determination of the order of the reaction of $\mathrm{PhBr}$ with hexylamine on the concentration of PhBr (Figure 8 (top)). Stock solutions of $\mathrm{Pd}(\mathrm{BINAP})_{2}$ in $\mathrm{C}_{6} \mathrm{D}_{6}(7.38 \mathrm{mM})$, 
BINAP in $\mathrm{C}_{6} \mathrm{D}_{6}(16.1 \mathrm{mM})$ and 1,3,5-trioxane (internal standard) in $\mathrm{C}_{6} \mathrm{D}_{6}(55.5 \mathrm{mM})$ were prepared. $\mathrm{NaOCEt}_{3}\left(13.5 \mathrm{mg}, 9.78 \times 10^{-5} \mathrm{~mol}\right)$ was weighed into an NMR tube with a screw cap lined with Teflon. $120 \mu \mathrm{L}$ of the stock solution of $\mathrm{Pd}(\mathrm{BINAP})_{2}\left(8.86 \times 10^{-7} \mathrm{~mol}\right), 100 \mu \mathrm{L}$ of the stock solution of BINAP $\left(1.61 \times 10^{-6} \mathrm{~mol}\right)$ and appropriate amount of $\mathrm{PhBr}\left(7.0 \times 10^{-5} \mathrm{~mol}-1.9 \times\right.$ $10^{-3} \mathrm{~mol}$ ) were added by syringe into the NMR tube. Appropriate amount of the stock solution of 1,3,5-trioxane was added to the NMR tube to have a total volume of $0.7 \mathrm{~mL}$. Hexylamine (1.2 $\mu \mathrm{L}, 9.18 \times 10^{-6} \mathrm{~mol}$ ) was added by syringe into the NMR tube just before inserting the tube into a preheated spectrometer probe.

Determination of the order of the reaction of $\mathrm{PhBr}$ with hexylamine on the concentration of BINAP (Figure 8 (bottom)). Stock solutions of $\mathrm{Pd}(\mathrm{BINAP})_{2}$ in $\mathrm{C}_{6} \mathrm{D}_{6}(7.38$ $\mathrm{mM})$, BINAP in $\mathrm{C}_{6} \mathrm{D}_{6}(16.1 \mathrm{mM})$ and 1,3,5-trioxane (internal standard) in $\mathrm{C}_{6} \mathrm{D}_{6}(55.5 \mathrm{mM})$ were prepared. $\mathrm{NaOCEt}_{3}\left(13.5 \mathrm{mg}, 9.78 \times 10^{-5} \mathrm{~mol}\right)$ was weighed into an NMR tube with a screw cap lined with Teflon. $120 \mu \mathrm{L}$ of the stock solution of $\operatorname{Pd}(\mathrm{BINAP})_{2}\left(8.86 \times 10^{-7} \mathrm{~mol}\right), 100-400 \mu \mathrm{L}$ of the stock solution of BINAP (1.61-6.44 x 10 $\left.0^{-6} \mathrm{~mol}\right)$ and $\operatorname{PhBr} 150 \mu \mathrm{L}\left(1.42 \times 10^{-3} \mathrm{~mol}\right)$ were added by syringe into the NMR tube. Appropriate amount of the stock solution of 1,3,5-trioxane was added to the NMR tube to have a total volume of $0.7 \mathrm{~mL}$. Hexylamine $\left(1.2 \mu \mathrm{L}, 9.18 \times 10^{-6}\right.$ mol) was added by syringe into the NMR tube just before inserting the tube into a preheated spectrometer probe.

\section{Experimental Section 2}

Materials. Anhydrous benzene was purchased from Aldrich and further dried by distilling from sodium benzophenone ketyl under nitrogen and stored in the glove box. (S)BINAP was purchased from Fluka and used without further purification. Bromo benzene was purchased form Aldrich and further purified by washing with concentrated $\mathrm{H}_{2} \mathrm{SO}_{4}$ and then with 
$\mathrm{NaHCO}_{3}$, followed by distillation from $\mathrm{CaH}_{2}$. It was then stored in a Schlenk over molecular seives. n-Hexyl amine was purchased from Aldrich and distilled from $\mathrm{CaH}_{2}$ and stored over molecular sieves. Sodium Amylate was purchased from Aldrich, stored in a glove box and small quantities were transferred to a round bottom flask prior to the reaction and dried under vacuum. $\operatorname{Pd}(\text { BINAP })_{2}$ was synthesized according to a reported procedure ${ }^{1}$ as given below.

Anhydrous $\mathrm{MeOH}$ was purchased from Aldrich and then degassed three times prior to use. Anhydrous toluene was purchased from Aldrich and further dried by distilling from sodium benzophenone ketyl under nitrogen. Anhydrous pentane from Aldrich, Sodium Hydroxide pellets from BDH, allyl palladium chloride dimer from Aldrich and (S)-BINAP from Fluka were used without further purification.

Catalyst Preparation. A solution of $\mathrm{NaOH}(2.4 \mathrm{~g}, 60 \mathrm{mmol})$ in degassed $\mathrm{MeOH}(50 \mathrm{~mL})$ was added to a yellow heterogeneous mixture of $[\mathrm{Pd}(\text { allyl }) \mathrm{Cl}]_{2}(0.4 \mathrm{~g}, 1.1 \mathrm{mmol})$ in $\mathrm{MeOH}(30$ $\mathrm{mL}$ ). The resulting clear solution was further degassed by three freeze-pump-thaw cycles and (S)-BINAP (2.7 g, $4.3 \mathrm{mmol})$ in $100 \mathrm{~mL}$ toluene was added to give a dark red solution. This reaction mixture was then degassed again ( 3 times) and stirred overnight at room temperature. The solvent was then removed in vacuo in a Schlenk line and then the solid washed with $\mathrm{MeOH}$ $(4 \times 25 \mathrm{~mL})$ and anhydrous pentane $(2 \times 10 \mathrm{~mL})$, and dried under vacuum to afford a dark red solid. (2.56g, $86.7 \%) .1 \mathrm{H}$ NMR (300 MHz, $\left.\mathrm{C}_{6} \mathrm{D}_{6}\right): \delta 8.35$ (br, s, 8H), 7.81 (br, d, 4H), 7.45 (br, $\mathrm{s}, 8 \mathrm{H}), 7.32-7.38(\mathrm{~m}, 8 \mathrm{H}), 7.17-7.28(\mathrm{~m}, 16 \mathrm{H}), 7.0-7.06(\mathrm{~m}, 4 \mathrm{H}), 6.8-6.87(\mathrm{~m}, 4 \mathrm{H}), 6.16(\mathrm{t}, 4 \mathrm{H})$ $5.85(\mathrm{t}, 4 \mathrm{H})$

Reaction procedure. Reactions were carried out in an Omnical Super CRC reaction calorimeter, which allows continuous monitoring of the instantaneous enthalpy balance around 
the vessel. The reaction vial was a $16 \mathrm{ml}$ septum-cap vial equipped with a shaft and stirring blade. Total reaction volume was $4.2-5 \mathrm{~mL}$. Reactions were carried out at $333 \mathrm{~K}$.

Reactions using $\mathrm{Pd}(\mathrm{BINAP})_{2}$ as catalyst were initiated by injecting a solution of $\operatorname{Pd}(\mathrm{BINAP})_{2}(10 \mathrm{mM})$ and $(\mathrm{S})$-BINAP $(2 \mathrm{mM})$ in benzene $(1.3 \mathrm{~mL})$ to a thermally equilibrated mixture of sodium amylate, bromobenzene, n-hexyl amine, benzene and tridecane (internal standard). In some experiments the reaction was initiated by injecting thermally equilibrated bromobenzene to a thermally equilibrated mixture of $\operatorname{Pd}(\mathrm{BINAP})_{2}(10 \mathrm{mM}),(\mathrm{S})-\mathrm{BINAP}(2 \mathrm{mM})$, sodium amylate, n-hexylamine, benzene and tridecane (internal standard). The two procedures gave the same rate profiles.

Reactions using $\mathrm{Pd}_{2} \mathrm{dba}_{3}$ /BINAP mixtures as catalyst were initiated by injecting identical aliquots of thermally equilibrated bromobenzene $\left([\mathrm{ArBr}]_{o}=0.17 \mathrm{M}\right)$ consecutively to a thermally equilibrated reaction mixture (ca. $60 \mathrm{~min}$ ) consisting of $19 \mathrm{mM}$ of $\mathrm{Pd}_{2}(\mathrm{dba})_{3}, 19 \mathrm{mM}$ of $(\mathrm{S})$ BINAP, 1.4 M of NaOt-Am, and 1.2 M of N-methylpiperazine (or n-hexyl amine).

\section{Crystallographic data for $[(\text { BINAP }) P d]_{2}(d b a)$}

\section{$\underline{\text { Data Collection }}$}

An orange blade crystal of $\mathrm{C}_{105} \mathrm{H}_{78} \mathrm{OP}_{4} \mathrm{Pd}_{2} \cdot 2 \mathrm{C}_{7} \mathrm{H}_{8}, \mathrm{C}_{119} \mathrm{H}_{94} \mathrm{OP}_{4} \mathrm{Pd}_{2}$, having approximate dimensions of $0.30 \times 0.25 \times 0.10 \mathrm{~mm}^{3}$ was mounted with epoxy cement on the tip of a fine glass fiber. All measurements were made on a Nonius KappaCCD diffractometer with graphite monochromated Mo-K $\alpha$ radiation. 
Cell constants and an orientation matrix for data collection corresponded to a primitive monoclinic cell with dimensions:

$$
\begin{array}{ll}
\mathrm{a}=9.7103(19) \AA & \alpha=90^{\circ} \\
\mathrm{b}=23.907(5) \AA & \beta=102.67(3) \mathrm{O} \\
\mathrm{c}=20.636(4) \AA & \gamma=90^{\circ} \\
\mathrm{V}=2285.1(8) \AA^{3} &
\end{array}
$$

For $\mathrm{Z}=2$ and F.W. = 1876.62, the calculated density is $1.333 \mathrm{~g} / \mathrm{cm}^{3}$. Based on a statistical analysis of intensity distribution, and the successful solution and refinement of the structure, the space group was determined to be $P 2_{1}(\# 4)$.

The data were collected at a temperature of $173(2) \mathrm{K}$ to a maximum $2 \theta$ value of $56.56^{\circ}$. Five omega scans consisting of 63, 63, 59, 37, and 41 data frames, respectively, were collected with a frame width of $1.2^{\circ}$ and a detector-to-crystal distance, Dx, of $35.0 \mathrm{~mm}$. Each frame was exposed twice (for the purpose of de-zingering) for a total of $42 \mathrm{~s}$. The data frames were processed and scaled using the DENZO software package. ${ }^{1}$

\section{Data Reduction}

A total of 20487 reflections were collected of which 20487 were unique and observed $\left(\mathrm{R}_{\text {int }}=0.00\right.$, Friedel pairs not merged $)$. The linear absorption coefficient, $\mu$, for Mo-K $\alpha$ radiation is $5.06 \mathrm{~cm}^{-1}$, and no absorption correction was applied. The data were corrected for Lorentz and polarization effects.

\section{$\underline{\text { Structure Solution and Refinement }}$}

The structure was solved by direct methods and expanded using Fourier techniques ${ }^{2}$. The non-hydrogen atoms were refined anisotropically, and hydrogen atoms were treated as idealized contributions. The final cycle of full-matrix least-squares refinement ${ }^{3}$ on $\mathrm{F}$ was based on 20487 observed reflections $(\mathrm{I}>2.00 \sigma(\mathrm{I}))$ and 1135 variable parameters and converged with unweighted and weighted agreement factors of:

$$
\begin{gathered}
\mathrm{R}=\Sigma\|\mathrm{Fo}|-| \mathrm{Fc}\| / \Sigma|\mathrm{Fo}|=0.0484 \\
\mathrm{R}_{\mathrm{W}}=\left\{\Sigma\left[\mathrm{w}\left(\mathrm{F}_{\mathrm{o}}{ }^{2}-\mathrm{F}_{\mathrm{c}}^{2}\right)^{2}\right] / \Sigma\left[\mathrm{w}\left(\mathrm{F}_{\mathrm{o}}^{2}\right)^{2}\right]\right\}^{1 / 2}=0.0831
\end{gathered}
$$

The maximum and minimum peaks on the final difference Fourier map corresponded to 0.434 and $-0.577 \mathrm{e}^{-} / \AA^{3}$ respectively. 
(1) Z. Otwinowski and W. Minor, "Processing of X-Ray Diffraction Data Collected in Oscillation Mode," Methods in Enzymology, vol. 276: Macromolecular Crystallography, part A, 307-326, 1997, C.W. Carter, Jr. \& R.M. Sweet, Eds., Academic Press.

(2) Acta Cryst. A46 (1990) 467-473

(3) Least Squares function minimized:

$$
\Sigma w\left(\mathrm{~F}_{\mathrm{o}}^{2}-\mathrm{F}_{\mathrm{c}}^{2}\right)^{2}
$$


Figure 1

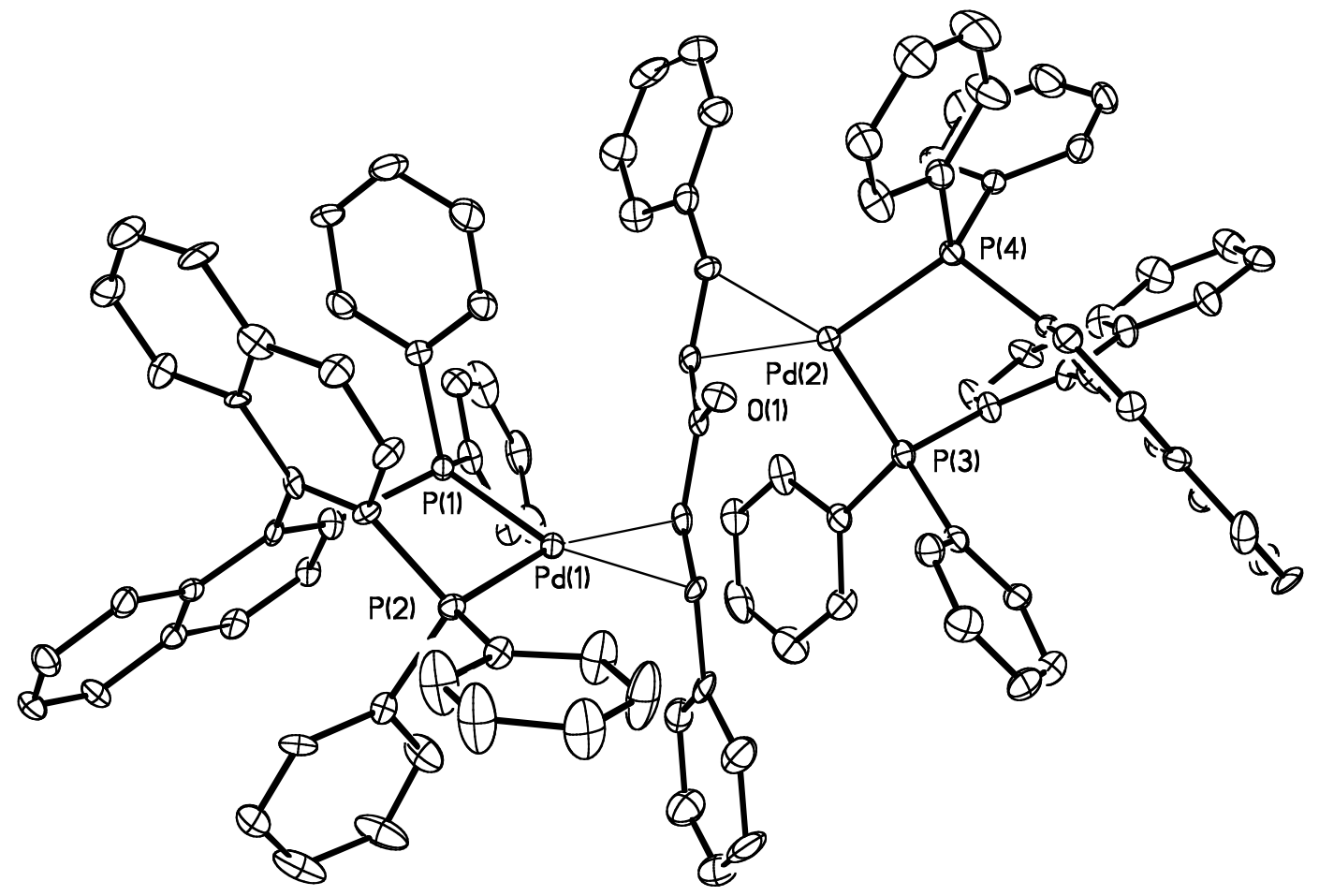

Figure 2

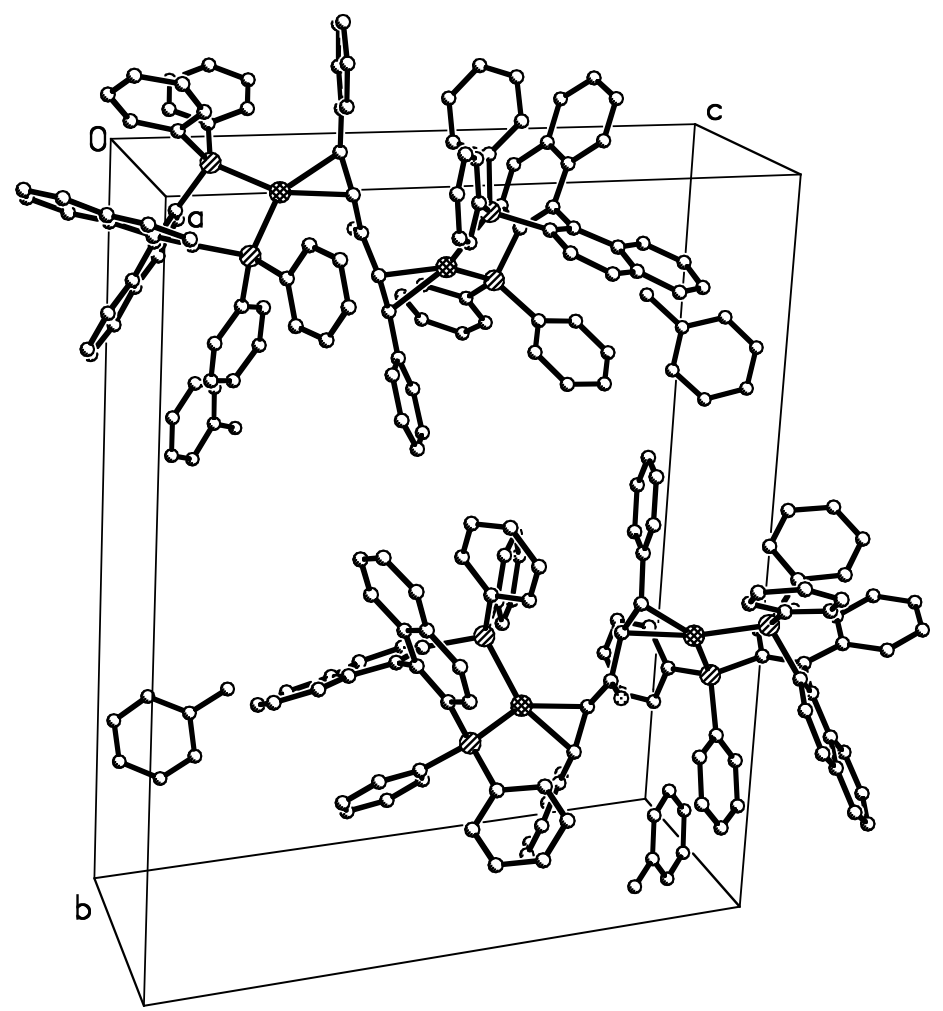


Figure 3

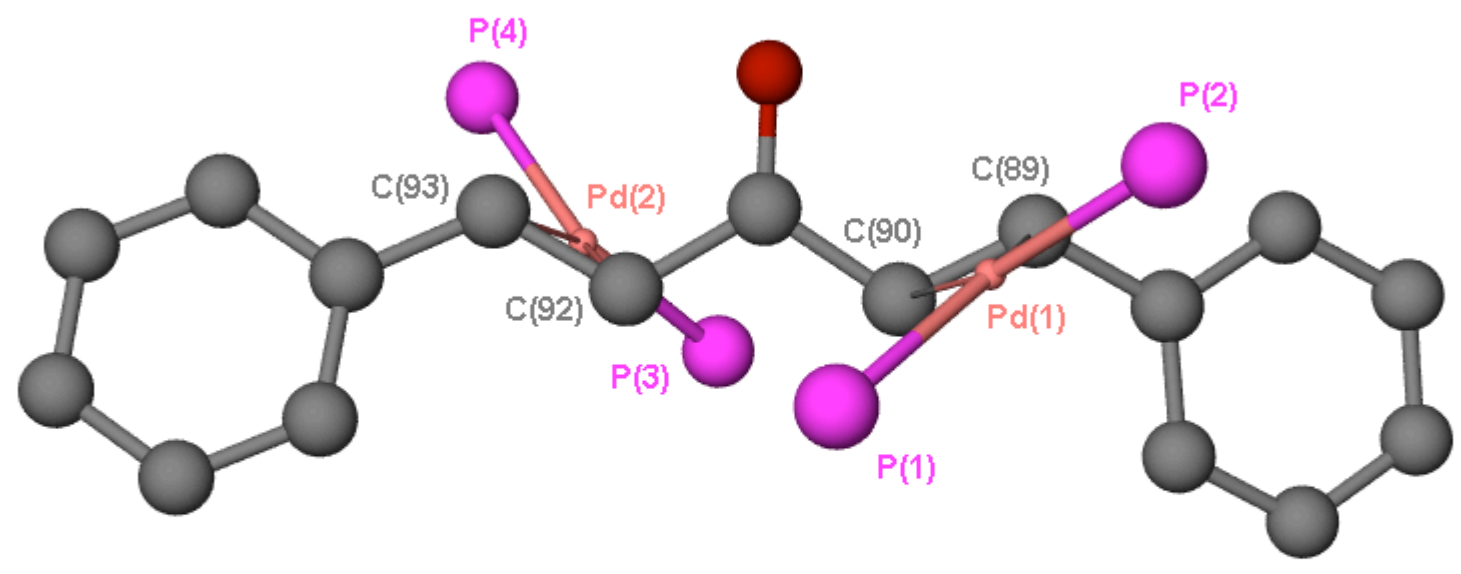


$\underline{\text { Packing diagram - View down the a-axis }}$

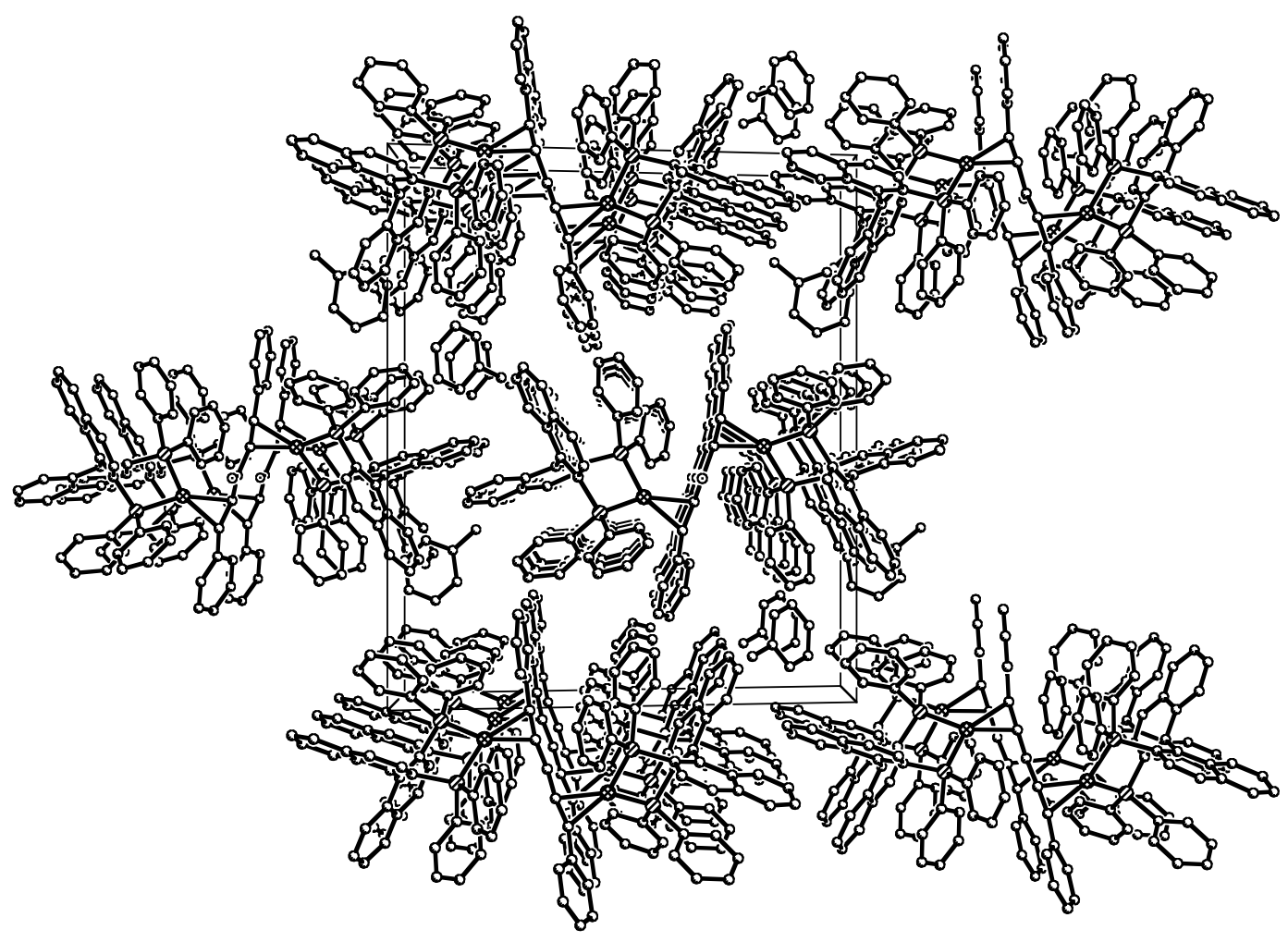




\section{Packing diagram - View down the b-axis}

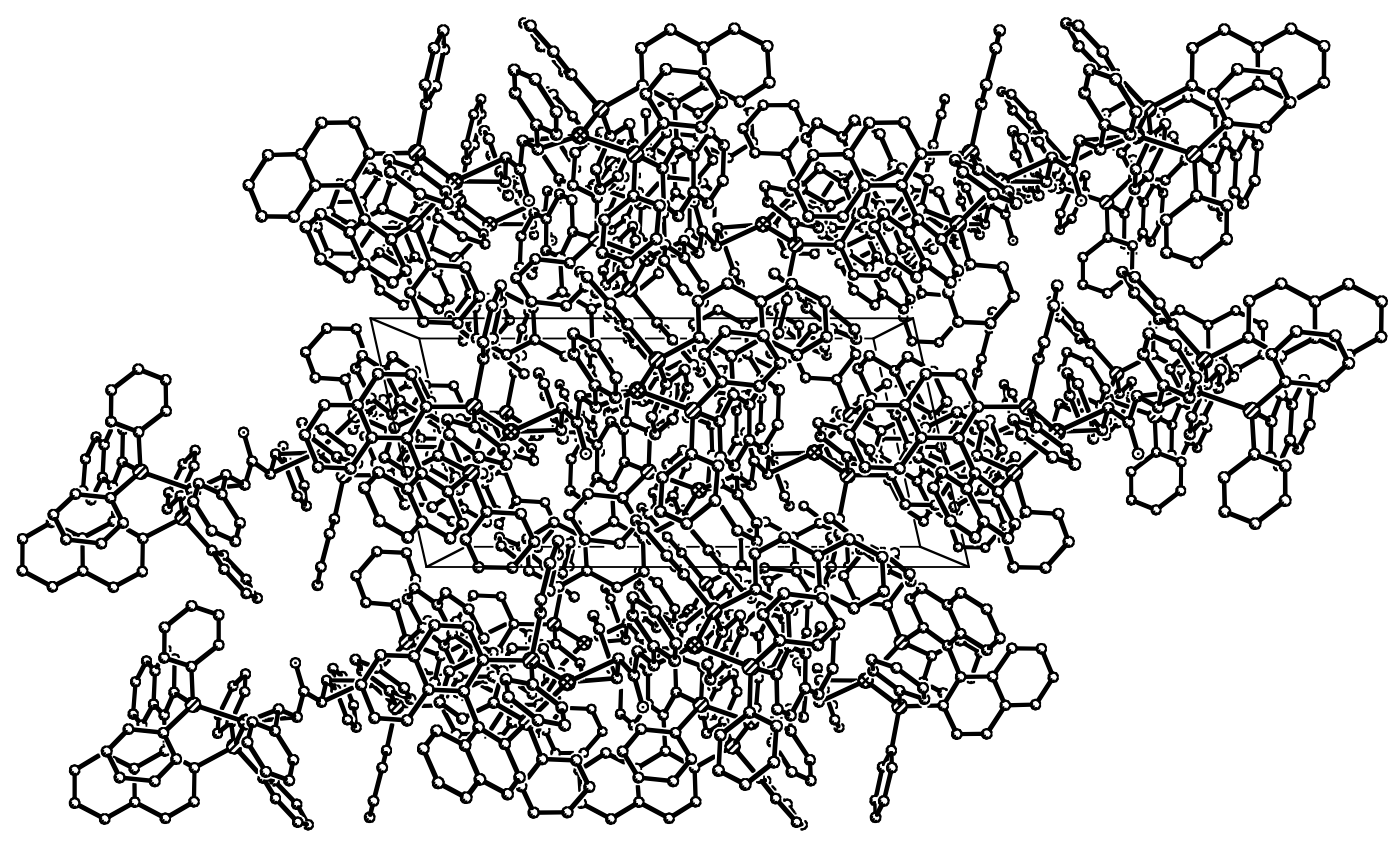


Packing diagram - View down the c-axis

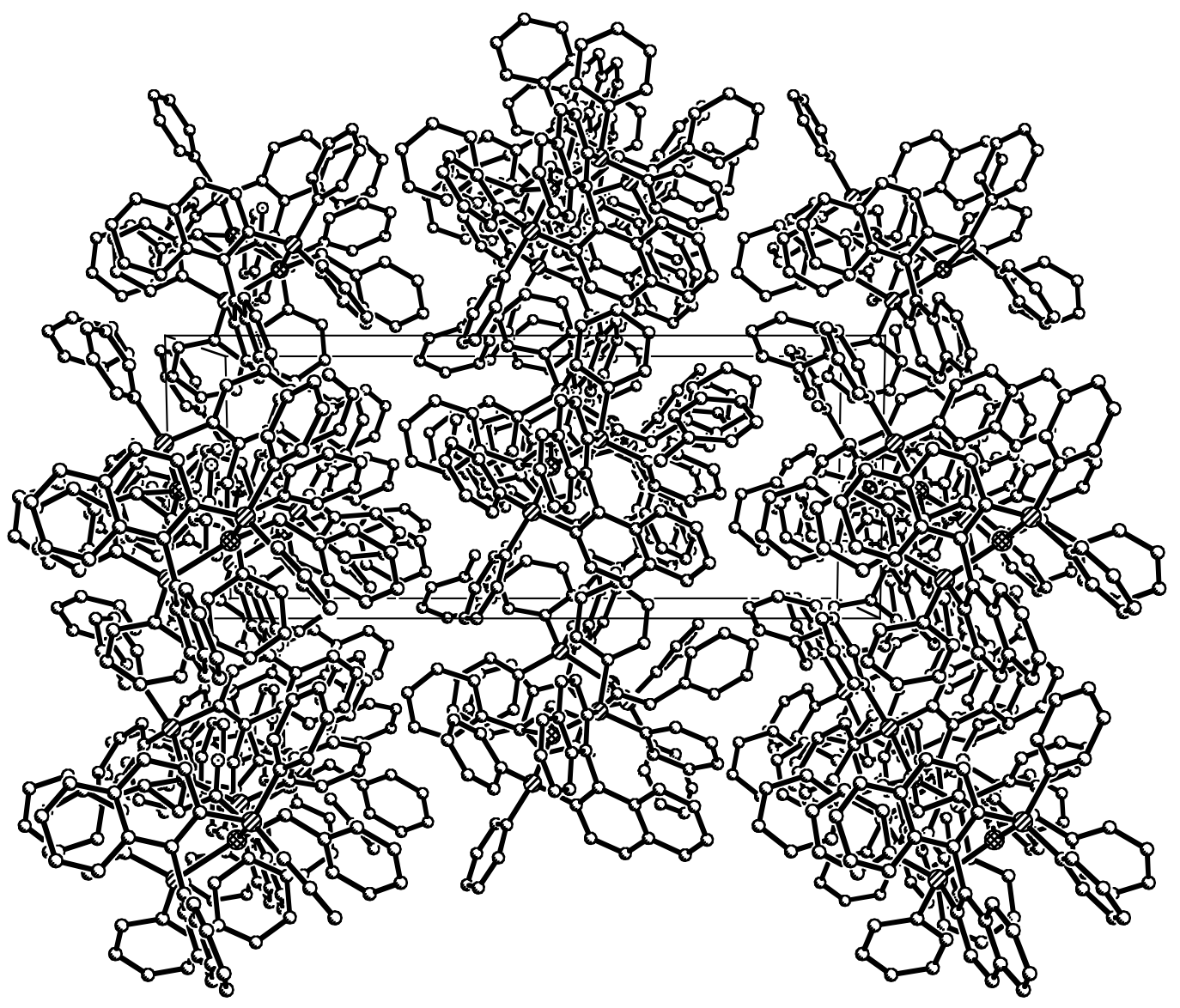


Table 1. Crystal data and structure refinement for $[(\mathrm{BINAP}) \mathrm{Pd}]_{2}(\mathrm{dba})$.

Identification code

Empirical formula

Formula weight

Temperature

Wavelength

Crystal system

Space group

Unit cell dimensions

Volume

Z

Density (calculated)

Absorption coefficient

$\mathrm{F}(000)$

Crystal size

Theta range for data collection

Index ranges

Reflections collected

Independent reflections

Completeness to theta $=28.28^{\circ}$

Absorption correction

Max. and min. transmission

Refinement method

Data / restraints / parameters

Goodness-of-fit on $\mathrm{F}^{2}$

Final $\mathrm{R}$ indices $[\mathrm{I}>2 \operatorname{sigma}(\mathrm{I})]$

$\mathrm{R}$ indices (all data)

Absolute structure parameter

Largest diff. peak and hole
$[(\mathrm{BINAP}) \mathrm{Pd}]_{2}(\mathrm{dba})$.

$\mathrm{C}_{119} \mathrm{H}_{94} \mathrm{O} \mathrm{P}_{4} \mathrm{Pd}_{2}$

1876.62

173(2) K

$0.71073 \AA$

Monoclinic

P2(1)

$\mathrm{a}=9.7103(19) \AA$

$\alpha=90^{\circ}$.

$\mathrm{b}=23.907(5) \AA$

$\beta=102.67(3)^{\circ}$.

$\mathrm{c}=20.636(4) \AA$

$\gamma=90^{\circ}$.

4674.1(16) $\AA^{3}$

2

$1.333 \mathrm{~g} / \mathrm{cm}^{3}$

$5.06 \mathrm{~cm}^{-1}$

1936

$0.30 \times 0.25 \times 0.10 \mathrm{~mm}^{3}$

2.17 to $28.28^{\circ}$.

$-12<=\mathrm{h}<=12,-31<=\mathrm{k}<=31,-27<=\mathrm{l}<=27$

20487

$20487[\mathrm{R}(\mathrm{int})=0.0000]$

$99.1 \%$

None

0.9512 and 0.8631

Full-matrix least-squares on $\mathrm{F}^{2}$

$20487 / 1 / 1135$

1.008

$\mathrm{R} 1=0.0484, \mathrm{wR}_{2}=0.0831$

$\mathrm{R} 1=0.0964, \mathrm{wR} 2=0.0960$

$-0.029(15)$

0.434 and -0.577 e. $\AA^{-3}$ 
Table 2. Atomic coordinates ( $\times 10^{4}$ ) and equivalent isotropic displacement parameters $\left(\AA^{2} \times 10^{3}\right)$ for $[(B I N A P) P d]_{2}(d b a) . U(e q)$ is defined as one third of the trace of the orthogonalized $U^{i j}$ tensor.

\begin{tabular}{|c|c|c|c|c|}
\hline & $\mathrm{x}$ & $\mathrm{y}$ & $\mathrm{z}$ & $\mathrm{U}(\mathrm{eq})$ \\
\hline $\operatorname{Pd}(1)$ & $14568(1)$ & $5342(1)$ & $7862(1)$ & 21(1) \\
\hline $\operatorname{Pd}(2)$ & $12949(1)$ & $6233(1)$ & $5368(1)$ & $22(1)$ \\
\hline $\mathrm{P}(1)$ & $13585(1)$ & $6036(1)$ & $8388(1)$ & $20(1)$ \\
\hline $\mathrm{P}(2)$ & $16212(1)$ & $5071(1)$ & $8781(1)$ & $22(1)$ \\
\hline $\mathrm{P}(3)$ & $11713(1)$ & $5459(1)$ & 4892(1) & $23(1)$ \\
\hline $\mathrm{P}(4)$ & $13754(1)$ & $6505(1)$ & $4454(1)$ & $23(1)$ \\
\hline $\mathrm{O}(1)$ & $15445(3)$ & $5903(1)$ & $6565(2)$ & $31(1)$ \\
\hline $\mathrm{C}(1)$ & 11705(4) & $6169(2)$ & $8018(2)$ & $26(1)$ \\
\hline$C(2)$ & $10868(5)$ & $5709(2)$ & 7791(2) & $34(1)$ \\
\hline$C(3)$ & $9438(5)$ & $5779(3)$ & 7494(2) & $44(1)$ \\
\hline$C(4)$ & $8857(5)$ & $6291(3)$ & 7404(2) & $50(2)$ \\
\hline$C(5)$ & $9689(5)$ & $6757(3)$ & 7616(3) & $50(2)$ \\
\hline$C(6)$ & $11099(5)$ & $6698(2)$ & $7925(2)$ & $36(1)$ \\
\hline$C(7)$ & 14393(4) & $6725(2)$ & $8410(2)$ & $24(1)$ \\
\hline$C(8)$ & $15272(5)$ & $6837(2)$ & $7979(2)$ & $33(1)$ \\
\hline $\mathrm{C}(9)$ & 15841(6) & $7370(2)$ & 7966(3) & $51(2)$ \\
\hline$C(10)$ & $15554(7)$ & $7777(2)$ & $8384(3)$ & $54(2)$ \\
\hline$C(11)$ & $14688(6)$ & $7670(2)$ & $8818(3)$ & $48(1)$ \\
\hline$C(12)$ & $14122(5)$ & $7146(2)$ & $8833(2)$ & $33(1)$ \\
\hline$C(13)$ & 13584(4) & $5870(2)$ & $9269(2)$ & $20(1)$ \\
\hline$C(14)$ & $14824(4)$ & $5844(2)$ & $9745(2)$ & $20(1)$ \\
\hline$C(15)$ & $14800(4)$ & $5645(2)$ & $10403(2)$ & $23(1)$ \\
\hline$C(16)$ & $16029(5)$ & $5615(2)$ & 10921(2) & $28(1)$ \\
\hline$C(17)$ & $15980(5)$ & $5409(2)$ & $11532(2)$ & $33(1)$ \\
\hline$C(18)$ & $14693(5)$ & $5217(2)$ & $11664(2)$ & $38(1)$ \\
\hline$C(19)$ & $13497(5)$ & $5243(2)$ & $11182(2)$ & $34(1)$ \\
\hline$C(20)$ & $13508(5)$ & $5461(2)$ & $10545(2)$ & $25(1)$ \\
\hline$C(21)$ & $12269(4)$ & $5496(2)$ & $10044(2)$ & $28(1)$ \\
\hline$C(22)$ & 12302(4) & $5696(2)$ & $9424(2)$ & $26(1)$ \\
\hline$C(23)$ & 16941(4) & $5708(2)$ & $9231(2)$ & $20(1)$ \\
\hline$C(24)$ & $16215(4)$ & $6029(2)$ & $9612(2)$ & $20(1)$ \\
\hline
\end{tabular}




\begin{tabular}{|c|c|c|c|c|}
\hline$C(25)$ & $16802(4)$ & $6547(2)$ & $9875(2)$ & $20(1)$ \\
\hline$C(26)$ & $16153(5)$ & $6887(2)$ & $10290(2)$ & $28(1)$ \\
\hline$C(27)$ & $16734(5)$ & $7380(2)$ & $10541(2)$ & $37(1)$ \\
\hline$C(28)$ & $17990(5)$ & $7570(2)$ & 10394(3) & $41(1)$ \\
\hline$C(29)$ & $18637(5)$ & $7268(2)$ & $9992(3)$ & $39(1)$ \\
\hline$C(30)$ & $18078(5)$ & $6747(2)$ & $9729(2)$ & $29(1)$ \\
\hline$C(31)$ & $18738(5)$ & $6419(2)$ & $9322(2)$ & $35(1)$ \\
\hline$C(32)$ & $18215(5)$ & 5914(2) & $9083(2)$ & $32(1)$ \\
\hline $\mathrm{C}(33)$ & $15532(4)$ & $4623(2)$ & $9354(2)$ & $25(1)$ \\
\hline$C(34)$ & $14315(5)$ & $4323(2)$ & $9085(3)$ & $41(1)$ \\
\hline$C(35)$ & $13757(5)$ & $3954(2)$ & $9471(3)$ & $60(2)$ \\
\hline$C(36)$ & $14382(6)$ & $3882(2)$ & 10121(3) & $56(2)$ \\
\hline$C(37)$ & $15600(6)$ & $4170(2)$ & $10402(3)$ & $49(2)$ \\
\hline $\mathrm{C}(38)$ & $16193(5)$ & $4533(2)$ & $10015(2)$ & $33(1)$ \\
\hline$C(39)$ & 17794(4) & $4690(2)$ & $8686(2)$ & $26(1)$ \\
\hline$C(40)$ & 18922(6) & $4575(3)$ & $9196(3)$ & $60(2)$ \\
\hline$C(41)$ & $20073(6)$ & $4281(3)$ & $9099(3)$ & $70(2)$ \\
\hline$C(42)$ & $20118(6)$ & 4095(3) & $8479(3)$ & $61(2)$ \\
\hline$C(43)$ & $19047(7)$ & $4228(3)$ & $7956(3)$ & $76(2)$ \\
\hline$C(44)$ & $17888(6)$ & $4519(3)$ & $8070(3)$ & $55(2)$ \\
\hline$C(45)$ & $12641(5)$ & $4811(2)$ & $4822(2)$ & $26(1)$ \\
\hline$C(46)$ & $12036(5)$ & $4370(2)$ & $4417(2)$ & $32(1)$ \\
\hline$C(47)$ & $12741(6)$ & $3870(2)$ & $4428(3)$ & $41(1)$ \\
\hline$C(48)$ & $14036(6)$ & $3800(2)$ & $4845(3)$ & $43(1)$ \\
\hline$C(49)$ & $14657(5)$ & $4235(2)$ & $5233(3)$ & $44(1)$ \\
\hline$C(50)$ & 13971(5) & $4740(2)$ & $5228(2)$ & $29(1)$ \\
\hline $\mathrm{C}(51)$ & 10273(4) & $5231(2)$ & $5289(2)$ & $30(1)$ \\
\hline$C(52)$ & 9992(5) & $4676(2)$ & $5408(2)$ & $40(1)$ \\
\hline$C(53)$ & $8926(6)$ & $4540(3)$ & $5736(3)$ & $60(2)$ \\
\hline$C(54)$ & $8136(5)$ & $4950(3)$ & $5941(3)$ & $50(2)$ \\
\hline$C(55)$ & $8407(5)$ & $5496(2)$ & $5831(2)$ & $41(1)$ \\
\hline$C(56)$ & $9486(5)$ & $5642(2)$ & $5518(2)$ & $34(1)$ \\
\hline$C(57)$ & $10825(4)$ & $5646(2)$ & $4034(2)$ & $24(1)$ \\
\hline $\mathrm{C}(58)$ & $11560(4)$ & $5694(2)$ & $3529(2)$ & $24(1)$ \\
\hline$C(59)$ & $10857(4)$ & $5899(2)$ & $2884(2)$ & $26(1)$ \\
\hline$C(60)$ & $11538(5)$ & $5925(2)$ & $2340(2)$ & $32(1)$ \\
\hline
\end{tabular}




\begin{tabular}{|c|c|c|c|c|}
\hline $\mathrm{C}(61)$ & $10846(5)$ & $6134(2)$ & $1744(2)$ & $40(1)$ \\
\hline$C(62)$ & $9477(5)$ & $6346(2)$ & $1649(2)$ & $44(1)$ \\
\hline$C(63)$ & 8793(5) & $6326(2)$ & $2166(2)$ & $42(1)$ \\
\hline$C(64)$ & $9451(5)$ & $6093(2)$ & $2786(2)$ & $33(1)$ \\
\hline$C(65)$ & $8747(5)$ & $6048(2)$ & $3311(2)$ & $37(1)$ \\
\hline$C(66)$ & $9398(5)$ & $5823(2)$ & $3908(2)$ & $35(1)$ \\
\hline$C(67)$ & 14157(4) & $5879(2)$ & $4010(2)$ & $21(1)$ \\
\hline$C(68)$ & $13099(4)$ & $5539(2)$ & $3643(2)$ & $23(1)$ \\
\hline$C(69)$ & $13472(5)$ & $5021(2)$ & $3373(2)$ & $26(1)$ \\
\hline$C(70)$ & $12466(5)$ & $4671(2)$ & $2966(2)$ & $33(1)$ \\
\hline$C(71)$ & $12852(6)$ & $4169(2)$ & $2734(2)$ & $41(1)$ \\
\hline$C(72)$ & $14265(6)$ & $3989(2)$ & $2895(2)$ & $43(1)$ \\
\hline$C(73)$ & $15249(5)$ & $4311(2)$ & $3280(2)$ & $39(1)$ \\
\hline$C(74)$ & $14901(5)$ & $4842(2)$ & $3519(2)$ & $28(1)$ \\
\hline$C(75)$ & $15923(5)$ & $5187(2)$ & $3905(2)$ & $31(1)$ \\
\hline$C(76)$ & $15574(5)$ & $5690(2)$ & $4134(2)$ & $28(1)$ \\
\hline$C(77)$ & $12650(5)$ & 6954(2) & $3840(2)$ & $27(1)$ \\
\hline$C(78)$ & $12813(5)$ & $7007(2)$ & $3183(2)$ & $30(1)$ \\
\hline$C(79)$ & $11957(5)$ & $7358(2)$ & $2743(2)$ & $39(1)$ \\
\hline $\mathrm{C}(80)$ & $10932(6)$ & $7655(2)$ & 2947(3) & $57(2)$ \\
\hline $\mathrm{C}(81)$ & 10757(7) & 7611(3) & $3598(3)$ & $67(2)$ \\
\hline$C(82)$ & $11605(5)$ & $7258(2)$ & $4036(3)$ & $44(1)$ \\
\hline$C(83)$ & 15451(4) & $6873(2)$ & $4659(2)$ & $28(1)$ \\
\hline$C(84)$ & $15889(5)$ & $7252(2)$ & $4241(3)$ & $53(2)$ \\
\hline$C(85)$ & $17219(6)$ & 7503(3) & $4433(3)$ & $68(2)$ \\
\hline$C(86)$ & 18104(6) & $7375(2)$ & $5023(3)$ & $50(2)$ \\
\hline $\mathrm{C}(87)$ & $17665(5)$ & $7000(2)$ & $5440(3)$ & $40(1)$ \\
\hline $\mathrm{C}(88)$ & $16356(5)$ & $6754(2)$ & $5267(2)$ & $34(1)$ \\
\hline$C(89)$ & $14490(5)$ & $4863(2)$ & $6957(2)$ & $23(1)$ \\
\hline $\mathrm{C}(90)$ & $13625(4)$ & $5336(2)$ & $6827(2)$ & $24(1)$ \\
\hline $\mathrm{C}(91)$ & 14193(4) & $5860(2)$ & $6599(2)$ & $24(1)$ \\
\hline $\mathrm{C}(92)$ & 13183(4) & $6318(2)$ & $6418(2)$ & $26(1)$ \\
\hline $\mathrm{C}(93)$ & 13621(4) & $6821(2)$ & $6169(2)$ & $24(1)$ \\
\hline$C(94)$ & $13968(5)$ & $4292(2)$ & $7024(2)$ & $29(1)$ \\
\hline $\mathrm{C}(95)$ & $14833(5)$ & $3832(2)$ & $7000(2)$ & $39(1)$ \\
\hline $\mathrm{C}(96)$ & $14373(7)$ & $3289(2)$ & 7077(3) & $54(2)$ \\
\hline
\end{tabular}




\begin{tabular}{|c|c|c|c|c|}
\hline $\mathrm{C}(97)$ & $13043(7)$ & $3198(2)$ & $7167(3)$ & $55(2)$ \\
\hline $\mathrm{C}(98)$ & $12143(6)$ & $3646(2)$ & 7179(3) & $55(2)$ \\
\hline$C(99)$ & $12602(5)$ & $4184(2)$ & $7107(2)$ & $40(1)$ \\
\hline$C(100)$ & $12820(5)$ & 7344(2) & 6091(2) & $27(1)$ \\
\hline$C(101)$ & $13280(6)$ & $7799(2)$ & $5767(3)$ & $42(1)$ \\
\hline$C(102)$ & $12563(7)$ & $8295(2)$ & $5673(3)$ & $54(2)$ \\
\hline$C(103)$ & $11349(6)$ & $8365(2)$ & 5912(3) & $52(2)$ \\
\hline$C(104)$ & $10883(6)$ & 7931(2) & $6247(3)$ & $49(1)$ \\
\hline$C(105)$ & $11612(5)$ & $7428(2)$ & $6332(2)$ & $39(1)$ \\
\hline$C(106)$ & $9409(8)$ & $3377(4)$ & 1816(4) & $80(2)$ \\
\hline$C(107)$ & $10275(9)$ & $3212(4)$ & $1416(4)$ & $91(2)$ \\
\hline$C(108)$ & $10723(8)$ & $3602(4)$ & $1025(3)$ & $83(2)$ \\
\hline$C(109)$ & $10355(8)$ & 4151(4) & 1061(3) & $88(3)$ \\
\hline$C(110)$ & $9487(8)$ & $4319(4)$ & $1485(4)$ & $84(2)$ \\
\hline$C(111)$ & $8993(7)$ & 3912(4) & 1873(3) & $86(2)$ \\
\hline$C(112)$ & $8087(10)$ & 4087(5) & $2327(4)$ & $150(5)$ \\
\hline$C(113)$ & $2817(6)$ & 7071(3) & $10567(4)$ & $65(2)$ \\
\hline$C(114)$ & 2191(7) & 7441(4) & $10082(4)$ & $77(2)$ \\
\hline$C(115)$ & $2303(7)$ & 7996(4) & $10181(4)$ & $79(2)$ \\
\hline$C(116)$ & $3043(7)$ & 8192(3) & $10782(4)$ & $71(2)$ \\
\hline$C(117)$ & $3705(6)$ & $7831(2)$ & $11270(3)$ & $55(2)$ \\
\hline$C(118)$ & $3592(6)$ & $7248(2)$ & $11175(3)$ & $51(2)$ \\
\hline C(119) & 4394(7) & $6859(3)$ & $11698(3)$ & $80(2)$ \\
\hline
\end{tabular}


Table 3. Bond lengths $[\AA]$ and angles $\left[{ }^{\circ}\right]$ for $[(\mathrm{BINAP}) \mathrm{Pd}]_{2}(\mathrm{dba})$.

\begin{tabular}{|c|c|c|c|}
\hline $\operatorname{Pd}(1)-C(90)$ & $2.132(3)$ & $C(13)-C(22)$ & $1.414(6)$ \\
\hline $\operatorname{Pd}(1)-C(89)$ & $2.179(4)$ & $C(14)-C(15)$ & $1.444(5)$ \\
\hline $\operatorname{Pd}(1)-P(2)$ & $2.2881(13)$ & $\mathrm{C}(14)-\mathrm{C}(24)$ & $1.503(6)$ \\
\hline $\operatorname{Pd}(1)-P(1)$ & $2.3009(11)$ & $C(15)-C(16)$ & $1.418(6)$ \\
\hline $\mathrm{Pd}(2)-\mathrm{C}(92)$ & $2.138(4)$ & $C(15)-C(20)$ & $1.419(6)$ \\
\hline $\mathrm{Pd}(2)-\mathrm{C}(93)$ & $2.158(4)$ & $C(16)-C(17)$ & $1.365(6)$ \\
\hline $\mathrm{Pd}(2)-\mathrm{P}(4)$ & $2.2885(12)$ & $C(17)-C(18)$ & $1.413(6)$ \\
\hline $\mathrm{Pd}(2)-\mathrm{P}(3)$ & $2.3057(12)$ & $C(18)-C(19)$ & $1.355(6)$ \\
\hline $\mathrm{P}(1)-\mathrm{C}(7)$ & $1.819(4)$ & $C(19)-C(20)$ & $1.416(6)$ \\
\hline $\mathrm{P}(1)-\mathrm{C}(1)$ & $1.845(4)$ & $C(20)-C(21)$ & $1.407(6)$ \\
\hline $\mathrm{P}(1)-\mathrm{C}(13)$ & $1.862(4)$ & $C(21)-C(22)$ & $1.372(6)$ \\
\hline $\mathrm{P}(2)-\mathrm{C}(33)$ & $1.824(4)$ & $C(23)-C(24)$ & $1.395(5)$ \\
\hline $\mathrm{P}(2)-\mathrm{C}(39)$ & $1.833(4)$ & $C(23)-C(32)$ & $1.426(6)$ \\
\hline $\mathrm{P}(2)-\mathrm{C}(23)$ & $1.842(4)$ & $C(24)-C(25)$ & $1.422(6)$ \\
\hline $\mathrm{P}(3)-\mathrm{C}(45)$ & $1.815(4)$ & $C(25)-C(30)$ & $1.421(6)$ \\
\hline $\mathrm{P}(3)-\mathrm{C}(57)$ & $1.844(4)$ & $C(25)-C(26)$ & $1.423(6)$ \\
\hline $\mathrm{P}(3)-\mathrm{C}(51)$ & $1.852(4)$ & $C(26)-C(27)$ & $1.361(6)$ \\
\hline $\mathrm{P}(4)-\mathrm{C}(77)$ & $1.820(4)$ & $\mathrm{C}(27)-\mathrm{C}(28)$ & $1.396(6)$ \\
\hline $\mathrm{P}(4)-\mathrm{C}(83)$ & $1.834(4)$ & $C(28)-C(29)$ & $1.354(7)$ \\
\hline $\mathrm{P}(4)-\mathrm{C}(67)$ & $1.842(4)$ & $C(29)-C(30)$ & $1.418(6)$ \\
\hline $\mathrm{O}(1)-\mathrm{C}(91)$ & $1.237(5)$ & $\mathrm{C}(30)-\mathrm{C}(31)$ & $1.402(6)$ \\
\hline$C(1)-C(2)$ & $1.385(6)$ & $\mathrm{C}(31)-\mathrm{C}(32)$ & $1.360(6)$ \\
\hline$C(1)-C(6)$ & $1.390(7)$ & $\mathrm{C}(33)-\mathrm{C}(38)$ & $1.390(6)$ \\
\hline $\mathrm{C}(2)-\mathrm{C}(3)$ & $1.400(7)$ & $\mathrm{C}(33)-\mathrm{C}(34)$ & $1.389(6)$ \\
\hline$C(3)-C(4)$ & $1.344(8)$ & $\mathrm{C}(34)-\mathrm{C}(35)$ & $1.377(7)$ \\
\hline$C(4)-C(5)$ & $1.388(8)$ & $\mathrm{C}(35)-\mathrm{C}(36)$ & $1.356(8)$ \\
\hline$C(5)-C(6)$ & $1.386(7)$ & $\mathrm{C}(36)-\mathrm{C}(37)$ & $1.381(8)$ \\
\hline $\mathrm{C}(7)-\mathrm{C}(8)$ & $1.387(6)$ & $\mathrm{C}(37)-\mathrm{C}(38)$ & $1.387(7)$ \\
\hline$C(7)-C(12)$ & $1.395(6)$ & $\mathrm{C}(39)-\mathrm{C}(44)$ & $1.358(6)$ \\
\hline $\mathrm{C}(8)-\mathrm{C}(9)$ & $1.390(7)$ & $C(39)-C(40)$ & $1.371(7)$ \\
\hline $\mathrm{C}(9)-\mathrm{C}(10)$ & $1.369(7)$ & $\mathrm{C}(40)-\mathrm{C}(41)$ & $1.371(7)$ \\
\hline$C(10)-C(11)$ & $1.381(8)$ & $C(41)-C(42)$ & $1.365(7)$ \\
\hline$C(11)-C(12)$ & $1.371(7)$ & $\mathrm{C}(42)-\mathrm{C}(43)$ & $1.363(8)$ \\
\hline$C(13)-C(14)$ & $1.378(6)$ & $C(43)-C(44)$ & $1.387(7)$ \\
\hline
\end{tabular}




\begin{tabular}{|c|c|c|c|}
\hline $\mathrm{C}(45)-\mathrm{C}(50)$ & $1.387(6)$ & $\mathrm{C}(77)-\mathrm{C}(78)$ & $1.404(6)$ \\
\hline$C(45)-C(46)$ & $1.392(6)$ & $C(78)-C(79)$ & $1.376(6)$ \\
\hline C(46)-C(47) & $1.376(7)$ & $\mathrm{C}(79)-\mathrm{C}(80)$ & $1.362(7)$ \\
\hline $\mathrm{C}(47)-\mathrm{C}(48)$ & $1.369(7)$ & $C(80)-C(81)$ & $1.395(7)$ \\
\hline C(48)-C(49) & $1.370(7)$ & $\mathrm{C}(81)-\mathrm{C}(82)$ & $1.371(7)$ \\
\hline C(49)-C(50) & $1.377(7)$ & $\mathrm{C}(83)-\mathrm{C}(84)$ & $1.381(6)$ \\
\hline $\mathrm{C}(51)-\mathrm{C}(52)$ & $1.385(6)$ & $C(83)-C(88)$ & $1.394(6)$ \\
\hline$C(51)-C(56)$ & $1.390(6)$ & $C(84)-C(85)$ & $1.401(7)$ \\
\hline$C(52)-C(53)$ & $1.394(6)$ & $C(85)-C(86)$ & $1.362(7)$ \\
\hline$C(53)-C(54)$ & $1.369(8)$ & $C(86)-C(87)$ & $1.373(7)$ \\
\hline$C(54)-C(55)$ & $1.360(7)$ & $\mathrm{C}(87)-\mathrm{C}(88)$ & $1.376(6)$ \\
\hline C(55)-C(56) & $1.391(6)$ & C(89)-C(90) & $1.399(6)$ \\
\hline $\mathrm{C}(57)-\mathrm{C}(58)$ & $1.391(6)$ & C(89)-C(94) & $1.474(6)$ \\
\hline C(57)-C(66) & $1.418(6)$ & $\mathrm{C}(90)-\mathrm{C}(91)$ & $1.486(6)$ \\
\hline C(58)-C(59) & $1.442(6)$ & $\mathrm{C}(91)-\mathrm{C}(92)$ & $1.463(6)$ \\
\hline C(58)-C(68) & $1.507(6)$ & $\mathrm{C}(92)-\mathrm{C}(93)$ & $1.409(6)$ \\
\hline C(59)-C(64) & $1.414(6)$ & $\mathrm{C}(93)-\mathrm{C}(100)$ & $1.463(6)$ \\
\hline C(59)-C(60) & $1.424(6)$ & $\mathrm{C}(94)-\mathrm{C}(95)$ & $1.392(6)$ \\
\hline $\mathrm{C}(60)-\mathrm{C}(61)$ & $1.359(6)$ & $C(94)-C(99)$ & $1.398(6)$ \\
\hline$C(61)-C(62)$ & $1.397(7)$ & $C(95)-C(96)$ & $1.392(7)$ \\
\hline $\mathrm{C}(62)-\mathrm{C}(63)$ & $1.376(6)$ & $\mathrm{C}(96)-\mathrm{C}(97)$ & $1.362(8)$ \\
\hline$C(63)-C(64)$ & $1.412(6)$ & C(97)-C(98) & $1.385(8)$ \\
\hline $\mathrm{C}(64)-\mathrm{C}(65)$ & $1.406(6)$ & $\mathrm{C}(98)-\mathrm{C}(99)$ & $1.381(7)$ \\
\hline$C(65)-C(66)$ & $1.366(6)$ & $C(100)-C(105)$ & $1.385(6)$ \\
\hline$C(67)-C(68)$ & $1.396(6)$ & $C(100)-C(101)$ & $1.400(7)$ \\
\hline $\mathrm{C}(67)-\mathrm{C}(76)$ & $1.417(6)$ & $\mathrm{C}(101)-\mathrm{C}(102)$ & $1.366(7)$ \\
\hline C(68)-C(69) & $1.436(6)$ & $C(102)-C(103)$ & $1.385(8)$ \\
\hline C(69)-C(70) & $1.414(6)$ & $C(103)-C(104)$ & $1.377(8)$ \\
\hline C(69)-C(74) & $1.420(6)$ & $C(104)-C(105)$ & $1.386(7)$ \\
\hline $\mathrm{C}(70)-\mathrm{C}(71)$ & $1.376(6)$ & $C(106)-C(111)$ & $1.354(10)$ \\
\hline$C(71)-C(72)$ & $1.406(7)$ & $C(106)-C(107)$ & $1.361(10)$ \\
\hline$C(72)-C(73)$ & $1.343(7)$ & $C(107)-C(108)$ & $1.365(10)$ \\
\hline$C(73)-C(74)$ & $1.429(6)$ & $\mathrm{C}(108)-\mathrm{C}(109)$ & $1.366(11)$ \\
\hline$C(74)-C(75)$ & $1.397(6)$ & $C(109)-C(110)$ & $1.401(10)$ \\
\hline$C(75)-C(76)$ & $1.362(6)$ & $\mathrm{C}(110)-\mathrm{C}(111)$ & $1.410(10)$ \\
\hline C(77)-C(82) & $1.378(6)$ & C(111)-C(112) & $1.479(10)$ \\
\hline
\end{tabular}




\begin{tabular}{|c|c|c|c|}
\hline$C(113)-C(114)$ & $1.372(10)$ & $\mathrm{C}(57)-\mathrm{P}(3)-\mathrm{Pd}(2)$ & $107.76(14)$ \\
\hline$C(113)-C(118)$ & $1.381(8)$ & $\mathrm{C}(51)-\mathrm{P}(3)-\mathrm{Pd}(2)$ & $114.89(14)$ \\
\hline$C(114)-C(115)$ & $1.344(10)$ & $\mathrm{C}(77)-\mathrm{P}(4)-\mathrm{C}(83)$ & $103.9(2)$ \\
\hline$C(115)-C(116)$ & $1.372(9)$ & $\mathrm{C}(77)-\mathrm{P}(4)-\mathrm{C}(67)$ & $106.82(19)$ \\
\hline$C(116)-C(117)$ & $1.373(8)$ & $\mathrm{C}(83)-\mathrm{P}(4)-\mathrm{C}(67)$ & $103.07(19)$ \\
\hline $\mathrm{C}(117)-\mathrm{C}(118)$ & $1.408(8)$ & $\mathrm{C}(77)-\mathrm{P}(4)-\mathrm{Pd}(2)$ & $119.07(14)$ \\
\hline \multirow[t]{2}{*}{$\mathrm{C}(118)-\mathrm{C}(119)$} & $1.506(8)$ & C(83)-P(4)-Pd(2) & $113.46(14)$ \\
\hline & & C(67)-P(4)-Pd(2) & $109.16(13)$ \\
\hline C(90)-Pd(1)-C(89) & $37.84(16)$ & $C(2)-C(1)-C(6)$ & $118.4(4)$ \\
\hline $\mathrm{C}(90)-\mathrm{Pd}(1)-\mathrm{P}(2)$ & $153.71(12)$ & $\mathrm{C}(2)-\mathrm{C}(1)-\mathrm{P}(1)$ & $117.2(4)$ \\
\hline C(89)-Pd(1)-P(2) & $116.64(12)$ & $\mathrm{C}(6)-\mathrm{C}(1)-\mathrm{P}(1)$ & $124.3(4)$ \\
\hline $\mathrm{C}(90)-\mathrm{Pd}(1)-\mathrm{P}(1)$ & $110.24(13)$ & $\mathrm{C}(1)-\mathrm{C}(2)-\mathrm{C}(3)$ & $120.4(5)$ \\
\hline C(89)-Pd(1)-P(1) & $147.48(12)$ & $C(4)-C(3)-C(2)$ & $121.0(5)$ \\
\hline $\mathrm{P}(2)-\mathrm{Pd}(1)-\mathrm{P}(1)$ & $95.74(4)$ & $\mathrm{C}(3)-\mathrm{C}(4)-\mathrm{C}(5)$ & $119.3(5)$ \\
\hline $\mathrm{C}(92)-\mathrm{Pd}(2)-\mathrm{C}(93)$ & $38.30(16)$ & $C(4)-C(5)-C(6)$ & $120.8(5)$ \\
\hline $\mathrm{C}(92)-\mathrm{Pd}(2)-\mathrm{P}(4)$ & $145.96(12)$ & $C(5)-C(6)-C(1)$ & $120.1(5)$ \\
\hline $\mathrm{C}(93)-\mathrm{Pd}(2)-\mathrm{P}(4)$ & $110.22(11)$ & $C(8)-C(7)-C(12)$ & $119.2(4)$ \\
\hline $\mathrm{C}(92)-\mathrm{Pd}(2)-\mathrm{P}(3)$ & $116.01(12)$ & $\mathrm{C}(8)-\mathrm{C}(7)-\mathrm{P}(1)$ & $118.8(3)$ \\
\hline $\mathrm{C}(93)-\mathrm{Pd}(2)-\mathrm{P}(3)$ & $153.01(11)$ & $\mathrm{C}(12)-\mathrm{C}(7)-\mathrm{P}(1)$ & $121.9(3)$ \\
\hline $\mathrm{P}(4)-\mathrm{Pd}(2)-\mathrm{P}(3)$ & $96.66(4)$ & $\mathrm{C}(9)-\mathrm{C}(8)-\mathrm{C}(7)$ & $119.5(4)$ \\
\hline$C(7)-P(1)-C(1)$ & $103.7(2)$ & $C(10)-C(9)-C(8)$ & $120.1(5)$ \\
\hline $\mathrm{C}(7)-\mathrm{P}(1)-\mathrm{C}(13)$ & $105.15(19)$ & $C(9)-C(10)-C(11)$ & $121.0(5)$ \\
\hline $\mathrm{C}(1)-\mathrm{P}(1)-\mathrm{C}(13)$ & $103.24(18)$ & $\mathrm{C}(12)-\mathrm{C}(11)-\mathrm{C}(10)$ & $119.2(5)$ \\
\hline $\mathrm{C}(7)-\mathrm{P}(1)-\mathrm{Pd}(1)$ & $116.30(14)$ & $C(11)-C(12)-C(7)$ & $120.9(5)$ \\
\hline $\mathrm{C}(1)-\mathrm{P}(1)-\mathrm{Pd}(1)$ & $113.79(14)$ & $\mathrm{C}(14)-\mathrm{C}(13)-\mathrm{C}(22)$ & $120.0(4)$ \\
\hline $\mathrm{C}(13)-\mathrm{P}(1)-\mathrm{Pd}(1)$ & $113.27(13)$ & $\mathrm{C}(14)-\mathrm{C}(13)-\mathrm{P}(1)$ & $121.2(3)$ \\
\hline $\mathrm{C}(33)-\mathrm{P}(2)-\mathrm{C}(39)$ & $102.01(19)$ & $\mathrm{C}(22)-\mathrm{C}(13)-\mathrm{P}(1)$ & $118.2(3)$ \\
\hline$C(33)-P(2)-C(23)$ & 108.19(19) & $C(13)-C(14)-C(15)$ & $119.6(4)$ \\
\hline $\mathrm{C}(39)-\mathrm{P}(2)-\mathrm{C}(23)$ & $103.08(19)$ & $C(13)-C(14)-C(24)$ & $122.4(3)$ \\
\hline $\mathrm{C}(33)-\mathrm{P}(2)-\mathrm{Pd}(1)$ & $114.69(14)$ & $C(15)-C(14)-C(24)$ & $118.0(3)$ \\
\hline$C(39)-P(2)-P d(1)$ & $120.08(15)$ & $C(16)-C(15)-C(20)$ & $117.7(4)$ \\
\hline $\mathrm{C}(23)-\mathrm{P}(2)-\mathrm{Pd}(1)$ & $107.77(13)$ & $C(16)-C(15)-C(14)$ & $122.7(4)$ \\
\hline $\mathrm{C}(45)-\mathrm{P}(3)-\mathrm{C}(57)$ & $105.5(2)$ & $\mathrm{C}(20)-\mathrm{C}(15)-\mathrm{C}(14)$ & $119.6(4)$ \\
\hline $\mathrm{C}(45)-\mathrm{P}(3)-\mathrm{C}(51)$ & $102.6(2)$ & $\mathrm{C}(17)-\mathrm{C}(16)-\mathrm{C}(15)$ & $121.3(4)$ \\
\hline $\mathrm{C}(57)-\mathrm{P}(3)-\mathrm{C}(51)$ & $104.92(19)$ & $C(16)-C(17)-C(18)$ & $120.5(4)$ \\
\hline $\mathrm{C}(45)-\mathrm{P}(3)-\mathrm{Pd}(2)$ & $119.87(15)$ & $\mathrm{C}(19)-\mathrm{C}(18)-\mathrm{C}(17)$ & $119.7(4)$ \\
\hline
\end{tabular}




\begin{tabular}{|c|c|c|c|}
\hline$C(18)-C(19)-C(20)$ & $121.3(4)$ & $C(42)-C(41)-C(40)$ & $119.9(5)$ \\
\hline $\mathrm{C}(21)-\mathrm{C}(20)-\mathrm{C}(19)$ & $121.7(4)$ & $\mathrm{C}(41)-\mathrm{C}(42)-\mathrm{C}(43)$ & $119.5(5)$ \\
\hline$C(21)-C(20)-C(15)$ & $118.8(4)$ & $C(42)-C(43)-C(44)$ & $119.2(5)$ \\
\hline$C(19)-C(20)-C(15)$ & $119.5(4)$ & $C(39)-C(44)-C(43)$ & $122.3(5)$ \\
\hline$C(22)-C(21)-C(20)$ & $121.0(4)$ & $C(50)-C(45)-C(46)$ & $119.0(4)$ \\
\hline $\mathrm{C}(21)-\mathrm{C}(22)-\mathrm{C}(13)$ & $121.0(4)$ & $\mathrm{C}(50)-\mathrm{C}(45)-\mathrm{P}(3)$ & $118.0(3)$ \\
\hline $\mathrm{C}(24)-\mathrm{C}(23)-\mathrm{C}(32)$ & $120.0(4)$ & $\mathrm{C}(46)-\mathrm{C}(45)-\mathrm{P}(3)$ & $122.8(3)$ \\
\hline $\mathrm{C}(24)-\mathrm{C}(23)-\mathrm{P}(2)$ & $123.5(3)$ & $\mathrm{C}(47)-\mathrm{C}(46)-\mathrm{C}(45)$ & $120.3(5)$ \\
\hline $\mathrm{C}(32)-\mathrm{C}(23)-\mathrm{P}(2)$ & $115.6(3)$ & $\mathrm{C}(48)-\mathrm{C}(47)-\mathrm{C}(46)$ & $120.2(5)$ \\
\hline$C(23)-C(24)-C(25)$ & $119.0(4)$ & $\mathrm{C}(47)-\mathrm{C}(48)-\mathrm{C}(49)$ & $120.0(5)$ \\
\hline $\mathrm{C}(23)-\mathrm{C}(24)-\mathrm{C}(14)$ & $121.8(4)$ & $\mathrm{C}(48)-\mathrm{C}(49)-\mathrm{C}(50)$ & $120.7(5)$ \\
\hline $\mathrm{C}(25)-\mathrm{C}(24)-\mathrm{C}(14)$ & 119.1(4) & $C(49)-C(50)-C(45)$ & $119.8(4)$ \\
\hline$C(30)-C(25)-C(26)$ & $117.3(4)$ & $\mathrm{C}(52)-\mathrm{C}(51)-\mathrm{C}(56)$ & $118.2(4)$ \\
\hline$C(30)-C(25)-C(24)$ & $120.5(4)$ & $\mathrm{C}(52)-\mathrm{C}(51)-\mathrm{P}(3)$ & $123.9(3)$ \\
\hline$C(26)-C(25)-C(24)$ & $122.3(4)$ & $\mathrm{C}(56)-\mathrm{C}(51)-\mathrm{P}(3)$ & $117.7(3)$ \\
\hline$C(27)-C(26)-C(25)$ & $121.5(4)$ & $\mathrm{C}(51)-\mathrm{C}(52)-\mathrm{C}(53)$ & $120.4(5)$ \\
\hline $\mathrm{C}(26)-\mathrm{C}(27)-\mathrm{C}(28)$ & $120.5(4)$ & $\mathrm{C}(54)-\mathrm{C}(53)-\mathrm{C}(52)$ & $120.6(5)$ \\
\hline$C(29)-C(28)-C(27)$ & $120.3(4)$ & $C(53)-C(54)-C(55)$ & $119.6(5)$ \\
\hline$C(28)-C(29)-C(30)$ & $120.9(4)$ & $\mathrm{C}(54)-\mathrm{C}(55)-\mathrm{C}(56)$ & $120.8(5)$ \\
\hline$C(31)-C(30)-C(25)$ & $118.3(4)$ & $C(55)-C(56)-C(51)$ & $120.4(5)$ \\
\hline$C(31)-C(30)-C(29)$ & $122.2(4)$ & $C(58)-C(57)-C(66)$ & $118.6(4)$ \\
\hline $\mathrm{C}(25)-\mathrm{C}(30)-\mathrm{C}(29)$ & $119.5(4)$ & $\mathrm{C}(58)-\mathrm{C}(57)-\mathrm{P}(3)$ & $122.0(3)$ \\
\hline $\mathrm{C}(32)-\mathrm{C}(31)-\mathrm{C}(30)$ & $122.0(4)$ & $\mathrm{C}(66)-\mathrm{C}(57)-\mathrm{P}(3)$ & $118.8(3)$ \\
\hline$C(31)-C(32)-C(23)$ & $120.1(4)$ & $\mathrm{C}(57)-\mathrm{C}(58)-\mathrm{C}(59)$ & $120.2(4)$ \\
\hline $\mathrm{C}(38)-\mathrm{C}(33)-\mathrm{C}(34)$ & $118.7(4)$ & $\mathrm{C}(57)-\mathrm{C}(58)-\mathrm{C}(68)$ & 121.1(4) \\
\hline C(38)-C(33)-P(2) & $124.9(3)$ & $C(59)-C(58)-C(68)$ & $118.7(4)$ \\
\hline C(34)-C(33)-P(2) & $116.2(3)$ & $C(64)-C(59)-C(60)$ & $118.2(4)$ \\
\hline$C(35)-C(34)-C(33)$ & $120.5(5)$ & $\mathrm{C}(64)-\mathrm{C}(59)-\mathrm{C}(58)$ & $119.4(4)$ \\
\hline$C(36)-C(35)-C(34)$ & $120.3(5)$ & $\mathrm{C}(60)-\mathrm{C}(59)-\mathrm{C}(58)$ & $122.4(4)$ \\
\hline$C(35)-C(36)-C(37)$ & $120.6(5)$ & $\mathrm{C}(61)-\mathrm{C}(60)-\mathrm{C}(59)$ & $120.5(4)$ \\
\hline $\mathrm{C}(38)-\mathrm{C}(37)-\mathrm{C}(36)$ & $119.7(5)$ & $\mathrm{C}(60)-\mathrm{C}(61)-\mathrm{C}(62)$ & $121.8(4)$ \\
\hline $\mathrm{C}(37)-\mathrm{C}(38)-\mathrm{C}(33)$ & $120.1(5)$ & $\mathrm{C}(63)-\mathrm{C}(62)-\mathrm{C}(61)$ & $119.0(4)$ \\
\hline C(44)-C(39)-C(40) & $116.9(4)$ & $C(62)-C(63)-C(64)$ & $121.0(4)$ \\
\hline C(44)-C(39)-P(2) & $118.7(4)$ & $\mathrm{C}(63)-\mathrm{C}(64)-\mathrm{C}(65)$ & $121.8(4)$ \\
\hline $\mathrm{C}(40)-\mathrm{C}(39)-\mathrm{P}(2)$ & $124.4(3)$ & $\mathrm{C}(63)-\mathrm{C}(64)-\mathrm{C}(59)$ & $119.4(4)$ \\
\hline$C(39)-C(40)-C(41)$ & $122.0(5)$ & $\mathrm{C}(65)-\mathrm{C}(64)-\mathrm{C}(59)$ & $118.8(4)$ \\
\hline
\end{tabular}




\begin{tabular}{|c|c|c|c|}
\hline$C(66)-C(65)-C(64)$ & $121.2(4)$ & C(90)-C(89)-C(94) & $124.3(4)$ \\
\hline $\mathrm{C}(65)-\mathrm{C}(66)-\mathrm{C}(57)$ & $121.6(4)$ & $\mathrm{C}(90)-\mathrm{C}(89)-\mathrm{Pd}(1)$ & $69.3(2)$ \\
\hline$C(68)-C(67)-C(76)$ & $118.8(4)$ & $\mathrm{C}(94)-\mathrm{C}(89)-\mathrm{Pd}(1)$ & $110.7(3)$ \\
\hline C(68)-C(67)-P(4) & $122.1(3)$ & $\mathrm{C}(89)-\mathrm{C}(90)-\mathrm{C}(91)$ & $119.6(4)$ \\
\hline C(76)-C(67)-P(4) & $118.2(3)$ & $\mathrm{C}(89)-\mathrm{C}(90)-\mathrm{Pd}(1)$ & $72.9(2)$ \\
\hline $\mathrm{C}(67)-\mathrm{C}(68)-\mathrm{C}(69)$ & 119.6(4) & $\mathrm{C}(91)-\mathrm{C}(90)-\mathrm{Pd}(1)$ & $101.7(3)$ \\
\hline $\mathrm{C}(67)-\mathrm{C}(68)-\mathrm{C}(58)$ & $121.9(4)$ & $\mathrm{O}(1)-\mathrm{C}(91)-\mathrm{C}(92)$ & $122.4(4)$ \\
\hline $\mathrm{C}(69)-\mathrm{C}(68)-\mathrm{C}(58)$ & $118.4(4)$ & $\mathrm{O}(1)-\mathrm{C}(91)-\mathrm{C}(90)$ & $121.7(4)$ \\
\hline $\mathrm{C}(70)-\mathrm{C}(69)-\mathrm{C}(74)$ & $117.6(4)$ & $\mathrm{C}(92)-\mathrm{C}(91)-\mathrm{C}(90)$ & $115.9(4)$ \\
\hline $\mathrm{C}(70)-\mathrm{C}(69)-\mathrm{C}(68)$ & $122.7(4)$ & $\mathrm{C}(93)-\mathrm{C}(92)-\mathrm{C}(91)$ & $119.5(4)$ \\
\hline C(74)-C(69)-C(68) & $119.7(4)$ & C(93)-C(92)-Pd(2) & $71.6(2)$ \\
\hline $\mathrm{C}(71)-\mathrm{C}(70)-\mathrm{C}(69)$ & $121.2(5)$ & $\mathrm{C}(91)-\mathrm{C}(92)-\mathrm{Pd}(2)$ & $95.9(2)$ \\
\hline $\mathrm{C}(70)-\mathrm{C}(71)-\mathrm{C}(72)$ & $120.7(5)$ & $\mathrm{C}(92)-\mathrm{C}(93)-\mathrm{C}(100)$ & $125.0(4)$ \\
\hline $\mathrm{C}(73)-\mathrm{C}(72)-\mathrm{C}(71)$ & $119.7(5)$ & $\mathrm{C}(92)-\mathrm{C}(93)-\mathrm{Pd}(2)$ & $70.1(2)$ \\
\hline $\mathrm{C}(72)-\mathrm{C}(73)-\mathrm{C}(74)$ & $121.4(5)$ & $\mathrm{C}(100)-\mathrm{C}(93)-\mathrm{Pd}(2)$ & 114.1(3) \\
\hline $\mathrm{C}(75)-\mathrm{C}(74)-\mathrm{C}(69)$ & $118.8(4)$ & $\mathrm{C}(95)-\mathrm{C}(94)-\mathrm{C}(99)$ & 117.1(4) \\
\hline$C(75)-C(74)-C(73)$ & $121.9(4)$ & $\mathrm{C}(95)-\mathrm{C}(94)-\mathrm{C}(89)$ & $120.4(4)$ \\
\hline $\mathrm{C}(69)-\mathrm{C}(74)-\mathrm{C}(73)$ & $119.3(4)$ & C(99)-C(94)-C(89) & $122.5(4)$ \\
\hline $\mathrm{C}(76)-\mathrm{C}(75)-\mathrm{C}(74)$ & $121.3(4)$ & $\mathrm{C}(96)-\mathrm{C}(95)-\mathrm{C}(94)$ & $121.4(5)$ \\
\hline $\mathrm{C}(75)-\mathrm{C}(76)-\mathrm{C}(67)$ & $121.6(4)$ & $\mathrm{C}(97)-\mathrm{C}(96)-\mathrm{C}(95)$ & $120.2(5)$ \\
\hline $\mathrm{C}(82)-\mathrm{C}(77)-\mathrm{C}(78)$ & $118.8(4)$ & $\mathrm{C}(96)-\mathrm{C}(97)-\mathrm{C}(98)$ & $120.0(5)$ \\
\hline C(82)-C(77)-P(4) & $117.8(3)$ & $\mathrm{C}(97)-\mathrm{C}(98)-\mathrm{C}(99)$ & $119.9(5)$ \\
\hline C(78)-C(77)-P(4) & $123.4(3)$ & C(98)-C(99)-C(94) & $121.5(5)$ \\
\hline $\mathrm{C}(79)-\mathrm{C}(78)-\mathrm{C}(77)$ & $120.9(4)$ & $C(105)-C(100)-C(101)$ & $116.3(4)$ \\
\hline $\mathrm{C}(80)-\mathrm{C}(79)-\mathrm{C}(78)$ & $119.3(5)$ & $C(105)-C(100)-C(93)$ & $123.8(4)$ \\
\hline $\mathrm{C}(79)-\mathrm{C}(80)-\mathrm{C}(81)$ & $120.6(5)$ & C(101)-C(100)-C(93) & $119.9(4)$ \\
\hline $\mathrm{C}(82)-\mathrm{C}(81)-\mathrm{C}(80)$ & $120.0(5)$ & $C(102)-C(101)-C(100)$ & $122.4(5)$ \\
\hline $\mathrm{C}(81)-\mathrm{C}(82)-\mathrm{C}(77)$ & $120.4(5)$ & C(101)-C(102)-C(103) & $120.1(5)$ \\
\hline $\mathrm{C}(84)-\mathrm{C}(83)-\mathrm{C}(88)$ & $118.5(4)$ & $C(102)-C(103)-C(104)$ & 119.1(5) \\
\hline C(84)-C(83)-P(4) & $123.7(4)$ & C(103)-C(104)-C(105) & $120.1(5)$ \\
\hline C(88)-C(83)-P(4) & $117.8(3)$ & $C(100)-C(105)-C(104)$ & $122.0(5)$ \\
\hline $\mathrm{C}(83)-\mathrm{C}(84)-\mathrm{C}(85)$ & $119.7(5)$ & C(111)-C(106)-C(107) & $124.2(8)$ \\
\hline$C(86)-C(85)-C(84)$ & $121.2(5)$ & C(108)-C(107)-C(106) & $118.8(8)$ \\
\hline $\mathrm{C}(85)-\mathrm{C}(86)-\mathrm{C}(87)$ & $119.0(5)$ & C(109)-C(108)-C(107) & $120.1(8)$ \\
\hline $\mathrm{C}(86)-\mathrm{C}(87)-\mathrm{C}(88)$ & $120.9(5)$ & C(108)-C(109)-C(110) & $120.8(7)$ \\
\hline $\mathrm{C}(87)-\mathrm{C}(88)-\mathrm{C}(83)$ & $120.6(4)$ & $\mathrm{C}(111)-\mathrm{C}(110)-\mathrm{C}(109)$ & $118.8(8)$ \\
\hline
\end{tabular}




$\begin{array}{ll}\mathrm{C}(106)-\mathrm{C}(111)-\mathrm{C}(110) & 117.3(7) \\ \mathrm{C}(106)-\mathrm{C}(111)-\mathrm{C}(112) & 123.5(8) \\ \mathrm{C}(110)-\mathrm{C}(111)-\mathrm{C}(112) & 119.1(9) \\ \mathrm{C}(114)-\mathrm{C}(113)-\mathrm{C}(118) & 122.1(7) \\ \mathrm{C}(115)-\mathrm{C}(114)-\mathrm{C}(113) & 121.1(7) \\ \mathrm{C}(114)-\mathrm{C}(115)-\mathrm{C}(116) & 118.9(7) \\ \mathrm{C}(115)-\mathrm{C}(116)-\mathrm{C}(117) & 121.1(7) \\ \mathrm{C}(116)-\mathrm{C}(117)-\mathrm{C}(118) & 120.6(6) \\ \mathrm{C}(113)-\mathrm{C}(118)-\mathrm{C}(117) & 116.1(6) \\ \mathrm{C}(113)-\mathrm{C}(118)-\mathrm{C}(119) & 123.8(6) \\ \mathrm{C}(117)-\mathrm{C}(118)-\mathrm{C}(119) & 119.9(6)\end{array}$


Table 4. Anisotropic displacement parameters $\left(\AA^{2} \times 10^{3}\right)$ for $[(\mathrm{BINAP}) \mathrm{Pd}]_{2}(\mathrm{dba})$. The anisotropic displacement factor exponent takes the form: $-2 \pi^{2}\left[h^{2} a^{* 2} U^{11}+\ldots+2 h k a^{*} b^{*} U^{12}\right]$

\begin{tabular}{|c|c|c|c|c|c|c|}
\hline & $\mathrm{U}^{11}$ & $\mathrm{U}^{22}$ & $\mathrm{U}^{33}$ & $\mathrm{U}^{23}$ & $\mathrm{U}^{13}$ & $\mathrm{U}^{12}$ \\
\hline $\operatorname{Pd}(1)$ & $23(1)$ & $21(1)$ & $19(1)$ & $-1(1)$ & $4(1)$ & 1(1) \\
\hline $\operatorname{Pd}(2)$ & $24(1)$ & $22(1)$ & $19(1)$ & $0(1)$ & $5(1)$ & $-1(1)$ \\
\hline $\mathrm{P}(1)$ & $20(1)$ & $22(1)$ & $19(1)$ & $-1(1)$ & $4(1)$ & $0(1)$ \\
\hline $\mathrm{P}(2)$ & $22(1)$ & $21(1)$ & $22(1)$ & $0(1)$ & $3(1)$ & $2(1)$ \\
\hline $\mathrm{P}(3)$ & $22(1)$ & $26(1)$ & 21(1) & $-2(1)$ & $5(1)$ & $-3(1)$ \\
\hline $\mathrm{P}(4)$ & $24(1)$ & $25(1)$ & 21(1) & $2(1)$ & $6(1)$ & $0(1)$ \\
\hline $\mathrm{O}(1)$ & $25(2)$ & $36(2)$ & $33(2)$ & $1(1)$ & $7(1)$ & $-3(1)$ \\
\hline$C(1)$ & $23(2)$ & $37(3)$ & $18(2)$ & $-3(2)$ & $5(2)$ & $7(2)$ \\
\hline$C(2)$ & $24(3)$ & $46(3)$ & $29(2)$ & $-5(2)$ & $1(2)$ & $-3(2)$ \\
\hline$C(3)$ & $33(3)$ & $66(4)$ & $31(3)$ & $-11(3)$ & $4(2)$ & $-10(3)$ \\
\hline$C(4)$ & $25(3)$ & $92(5)$ & $30(3)$ & $-4(3)$ & $3(2)$ & $10(3)$ \\
\hline$C(5)$ & $38(3)$ & $68(4)$ & $44(3)$ & $10(3)$ & $8(3)$ & $17(3)$ \\
\hline$C(6)$ & $27(3)$ & $44(3)$ & $36(3)$ & $2(2)$ & $3(2)$ & $6(2)$ \\
\hline$C(7)$ & $25(2)$ & $22(2)$ & $22(2)$ & $1(2)$ & $0(2)$ & $-1(2)$ \\
\hline$C(8)$ & $37(3)$ & $32(3)$ & $30(3)$ & $2(2)$ & $10(2)$ & $-8(2)$ \\
\hline C(9) & $67(4)$ & $44(3)$ & $44(3)$ & $2(3)$ & $20(3)$ & $-26(3)$ \\
\hline$C(10)$ & $83(4)$ & $28(3)$ & $48(3)$ & $4(3)$ & $7(3)$ & $-21(3)$ \\
\hline $\mathrm{C}(11)$ & $80(4)$ & $25(3)$ & $39(3)$ & $-4(2)$ & $12(3)$ & $-9(3)$ \\
\hline$C(12)$ & $42(3)$ & $33(3)$ & $25(2)$ & $2(2)$ & $7(2)$ & $1(2)$ \\
\hline$C(13)$ & $20(2)$ & $18(2)$ & $21(2)$ & $-1(2)$ & $5(2)$ & $1(2)$ \\
\hline$C(14)$ & $24(2)$ & $20(2)$ & $16(2)$ & $-5(2)$ & $4(2)$ & $0(2)$ \\
\hline$C(15)$ & $30(2)$ & $16(2)$ & $20(2)$ & $0(2)$ & $3(2)$ & $1(2)$ \\
\hline$C(16)$ & $28(3)$ & $29(3)$ & $26(2)$ & $-3(2)$ & $2(2)$ & $1(2)$ \\
\hline$C(17)$ & $39(3)$ & $32(3)$ & $28(2)$ & $3(2)$ & $2(2)$ & $3(2)$ \\
\hline$C(18)$ & $55(3)$ & $38(3)$ & $21(2)$ & $7(2)$ & $11(2)$ & $-3(2)$ \\
\hline$C(19)$ & $43(3)$ & $33(3)$ & $31(2)$ & $4(2)$ & $15(2)$ & $-6(2)$ \\
\hline$C(20)$ & $31(2)$ & $18(3)$ & $28(2)$ & $-2(2)$ & $14(2)$ & $-2(2)$ \\
\hline $\mathrm{C}(21)$ & $23(2)$ & $32(3)$ & $30(2)$ & $-1(2)$ & $11(2)$ & $-8(2)$ \\
\hline$C(22)$ & $22(2)$ & $28(3)$ & $27(2)$ & $-3(2)$ & $7(2)$ & $-3(2)$ \\
\hline$C(23)$ & $18(2)$ & $21(2)$ & $19(2)$ & $-1(2)$ & $0(2)$ & $1(2)$ \\
\hline $\mathrm{C}(24)$ & $17(2)$ & $24(2)$ & $17(2)$ & $7(2)$ & $-3(2)$ & $0(2)$ \\
\hline
\end{tabular}




\begin{tabular}{|c|c|c|c|c|c|c|}
\hline$C(25)$ & $20(2)$ & $20(2)$ & $18(2)$ & $-1(2)$ & $-1(2)$ & $-1(2)$ \\
\hline$C(26)$ & $26(2)$ & $23(3)$ & $33(2)$ & $-3(2)$ & $3(2)$ & $-2(2)$ \\
\hline$C(27)$ & $40(3)$ & $33(3)$ & $38(3)$ & $-9(2)$ & $11(2)$ & $-2(2)$ \\
\hline$C(28)$ & $38(3)$ & $27(3)$ & $55(3)$ & $-15(2)$ & $5(3)$ & $-9(2)$ \\
\hline $\mathrm{C}(29)$ & $30(3)$ & $33(3)$ & $56(3)$ & $-5(2)$ & $15(3)$ & $-12(2)$ \\
\hline$C(30)$ & $24(2)$ & $27(3)$ & $35(3)$ & $5(2)$ & $5(2)$ & $-1(2)$ \\
\hline$C(31)$ & $28(3)$ & $39(3)$ & $43(3)$ & $-11(2)$ & $18(2)$ & $-10(2)$ \\
\hline$C(32)$ & $22(3)$ & $39(3)$ & $38(3)$ & $-8(2)$ & $12(2)$ & $-2(2)$ \\
\hline$C(33)$ & $23(2)$ & $19(2)$ & $33(2)$ & $2(2)$ & $5(2)$ & $3(2)$ \\
\hline$C(34)$ & $27(3)$ & 41(3) & $53(3)$ & $20(3)$ & $4(2)$ & $-1(2)$ \\
\hline$C(35)$ & $28(3)$ & $52(4)$ & $95(5)$ & 41(3) & $6(3)$ & $-5(3)$ \\
\hline$C(36)$ & $43(3)$ & $49(4)$ & $83(5)$ & $42(3)$ & $25(3)$ & $10(3)$ \\
\hline$C(37)$ & 70(4) & $39(3)$ & $43(3)$ & 19(3) & 21(3) & $17(3)$ \\
\hline$C(38)$ & $40(3)$ & $21(2)$ & $37(3)$ & $7(2)$ & $7(2)$ & $0(2)$ \\
\hline$C(39)$ & $26(2)$ & $24(2)$ & $27(2)$ & $-2(2)$ & $7(2)$ & 1(2) \\
\hline $\mathrm{C}(40)$ & $52(4)$ & $92(5)$ & $33(3)$ & $-5(3)$ & 4(3) & $39(3)$ \\
\hline $\mathrm{C}(41)$ & $51(4)$ & $113(6)$ & $39(3)$ & $-7(3)$ & $-4(3)$ & $49(4)$ \\
\hline$C(42)$ & $46(4)$ & $84(5)$ & $54(4)$ & $-7(3)$ & $10(3)$ & $37(3)$ \\
\hline$C(43)$ & 64(4) & $119(6)$ & $38(3)$ & $-27(4)$ & $-3(3)$ & $56(4)$ \\
\hline$C(44)$ & 44(3) & $77(4)$ & $37(3)$ & $-15(3)$ & $-7(3)$ & $27(3)$ \\
\hline$C(45)$ & $31(3)$ & $27(3)$ & $22(2)$ & $-1(2)$ & $11(2)$ & $-3(2)$ \\
\hline$C(46)$ & $38(3)$ & $25(3)$ & $33(3)$ & $-5(2)$ & $9(2)$ & $-9(2)$ \\
\hline $\mathrm{C}(47)$ & $47(3)$ & $36(3)$ & $44(3)$ & $-10(2)$ & $15(3)$ & $-4(3)$ \\
\hline C(48) & $50(3)$ & $31(3)$ & $53(3)$ & $-1(3)$ & $23(3)$ & $11(3)$ \\
\hline $\mathrm{C}(49)$ & $39(3)$ & $47(3)$ & $45(3)$ & $4(3)$ & $5(3)$ & $10(3)$ \\
\hline $\mathrm{C}(50)$ & 26(3) & $33(3)$ & $26(2)$ & $-4(2)$ & $3(2)$ & $1(2)$ \\
\hline $\mathrm{C}(51)$ & $30(2)$ & $36(3)$ & $22(2)$ & $1(2)$ & $4(2)$ & $-2(2)$ \\
\hline$C(52)$ & 44(3) & $33(3)$ & $51(3)$ & $-6(2)$ & $30(3)$ & $-9(2)$ \\
\hline$C(53)$ & 68(4) & $57(4)$ & 71(4) & $-3(3)$ & $49(4)$ & $-25(3)$ \\
\hline$C(54)$ & $38(3)$ & $70(4)$ & $49(3)$ & $-9(3)$ & $26(3)$ & $-17(3)$ \\
\hline$C(55)$ & 28(3) & $63(4)$ & $35(3)$ & $-3(2)$ & $12(2)$ & $7(2)$ \\
\hline$C(56)$ & $37(3)$ & $32(3)$ & $33(3)$ & $-2(2)$ & $10(2)$ & $1(2)$ \\
\hline $\mathrm{C}(57)$ & $23(2)$ & $26(2)$ & $24(2)$ & $1(2)$ & $5(2)$ & $-3(2)$ \\
\hline$C(58)$ & $24(2)$ & $24(3)$ & $22(2)$ & $-2(2)$ & $1(2)$ & $-1(2)$ \\
\hline$C(59)$ & $24(2)$ & $25(2)$ & $26(2)$ & $0(2)$ & $-1(2)$ & $-5(2)$ \\
\hline$C(60)$ & $30(3)$ & $40(3)$ & $23(2)$ & $2(2)$ & $2(2)$ & $0(2)$ \\
\hline
\end{tabular}




\begin{tabular}{|c|c|c|c|c|c|c|}
\hline$C(61)$ & $40(3)$ & $51(4)$ & $25(2)$ & $7(2)$ & $1(2)$ & $-4(3)$ \\
\hline$C(62)$ & $47(3)$ & $52(4)$ & $26(2)$ & $11(2)$ & $-8(2)$ & 2(3) \\
\hline$C(63)$ & $26(2)$ & $58(4)$ & $37(3)$ & $10(3)$ & $-7(2)$ & $7(2)$ \\
\hline$C(64)$ & $26(2)$ & $38(3)$ & $29(2)$ & $-2(2)$ & $-2(2)$ & $-4(2)$ \\
\hline$C(65)$ & $17(2)$ & $54(3)$ & $36(3)$ & $3(2)$ & $-2(2)$ & $1(2)$ \\
\hline$C(66)$ & $27(3)$ & $51(3)$ & $28(2)$ & $3(2)$ & $7(2)$ & $0(2)$ \\
\hline$C(67)$ & $20(2)$ & $27(2)$ & $15(2)$ & $4(2)$ & $4(2)$ & $1(2)$ \\
\hline$C(68)$ & $20(2)$ & $30(3)$ & $19(2)$ & $7(2)$ & $6(2)$ & $4(2)$ \\
\hline$C(69)$ & $33(3)$ & $29(3)$ & $16(2)$ & $1(2)$ & $5(2)$ & $1(2)$ \\
\hline$C(70)$ & $36(3)$ & $34(3)$ & $26(2)$ & $-4(2)$ & $0(2)$ & $-4(2)$ \\
\hline$C(71)$ & $51(3)$ & $40(3)$ & $32(3)$ & $-12(2)$ & $6(2)$ & $-7(3)$ \\
\hline$C(72)$ & $61(4)$ & $33(3)$ & $36(3)$ & $-11(2)$ & $9(3)$ & 4(3) \\
\hline$C(73)$ & $43(3)$ & $47(3)$ & $31(3)$ & $1(2)$ & $14(2)$ & $13(3)$ \\
\hline$C(74)$ & $33(3)$ & $27(3)$ & $27(2)$ & $1(2)$ & $13(2)$ & $4(2)$ \\
\hline$C(75)$ & $25(2)$ & $38(3)$ & $33(2)$ & $4(2)$ & $10(2)$ & $7(2)$ \\
\hline$C(76)$ & $25(2)$ & $34(3)$ & $24(2)$ & $2(2)$ & $5(2)$ & $0(2)$ \\
\hline$C(77)$ & $33(3)$ & $20(2)$ & $29(2)$ & $0(2)$ & $10(2)$ & $1(2)$ \\
\hline$C(78)$ & $27(2)$ & $32(3)$ & $32(2)$ & $5(2)$ & $9(2)$ & $3(2)$ \\
\hline$C(79)$ & $51(3)$ & $39(3)$ & $27(3)$ & $10(2)$ & $8(2)$ & $5(3)$ \\
\hline$C(80)$ & $57(4)$ & $65(4)$ & $48(3)$ & $25(3)$ & $9(3)$ & $29(3)$ \\
\hline$C(81)$ & $75(4)$ & $72(5)$ & $60(4)$ & $15(3)$ & $31(3)$ & $45(4)$ \\
\hline$C(82)$ & $52(3)$ & $45(3)$ & $40(3)$ & $13(3)$ & $20(3)$ & $19(3)$ \\
\hline$C(83)$ & $29(2)$ & $26(3)$ & $29(2)$ & $-3(2)$ & $8(2)$ & $-3(2)$ \\
\hline$C(84)$ & $43(3)$ & $67(4)$ & $42(3)$ & $15(3)$ & $-4(3)$ & $-28(3)$ \\
\hline$C(85)$ & $58(4)$ & $85(5)$ & $57(4)$ & $16(4)$ & $3(3)$ & $-41(4)$ \\
\hline$C(86)$ & $38(3)$ & $62(4)$ & $47(3)$ & $-3(3)$ & $0(3)$ & $-22(3)$ \\
\hline$C(87)$ & $33(3)$ & $42(3)$ & $42(3)$ & $-7(2)$ & $1(2)$ & $-5(2)$ \\
\hline $\mathrm{C}(88)$ & $26(3)$ & $43(3)$ & $33(3)$ & $1(2)$ & $6(2)$ & $0(2)$ \\
\hline C(89) & $25(2)$ & $23(2)$ & $21(2)$ & $-3(2)$ & $6(2)$ & $3(2)$ \\
\hline$C(90)$ & $26(2)$ & $32(2)$ & $11(2)$ & $-6(2)$ & $-1(2)$ & $-4(2)$ \\
\hline $\mathrm{C}(91)$ & $27(3)$ & $31(3)$ & $11(2)$ & $-6(2)$ & $1(2)$ & $-7(2)$ \\
\hline $\mathrm{C}(92)$ & $33(2)$ & $27(3)$ & $17(2)$ & $-3(2)$ & $5(2)$ & $3(2)$ \\
\hline $\mathrm{C}(93)$ & $22(2)$ & $27(3)$ & $23(2)$ & $-4(2)$ & $4(2)$ & $-2(2)$ \\
\hline $\mathrm{C}(94)$ & $37(3)$ & $28(3)$ & $21(2)$ & $-12(2)$ & $4(2)$ & $1(2)$ \\
\hline$C(95)$ & $40(3)$ & $33(3)$ & $43(3)$ & $-7(2)$ & $3(2)$ & $1(2)$ \\
\hline $\mathrm{C}(96)$ & $78(4)$ & $25(3)$ & $56(4)$ & $-7(3)$ & $6(3)$ & $7(3)$ \\
\hline
\end{tabular}




\begin{tabular}{|c|c|c|c|c|c|c|}
\hline $\mathrm{C}(97)$ & $79(4)$ & $28(3)$ & 61(4) & $-5(3)$ & $17(3)$ & $-20(3)$ \\
\hline $\mathrm{C}(98)$ & $64(4)$ & $45(4)$ & $61(4)$ & $-12(3)$ & $27(3)$ & $-18(3)$ \\
\hline C(99) & $51(3)$ & $31(3)$ & $43(3)$ & $-8(2)$ & $18(3)$ & $-6(3)$ \\
\hline$C(100)$ & $29(3)$ & $24(3)$ & $27(2)$ & $-6(2)$ & $4(2)$ & $-4(2)$ \\
\hline$C(101)$ & $47(3)$ & $29(3)$ & $52(3)$ & $-5(2)$ & $16(3)$ & $-2(2)$ \\
\hline$C(102)$ & $72(4)$ & $27(3)$ & 61(4) & $3(3)$ & $15(3)$ & $3(3)$ \\
\hline$C(103)$ & $54(4)$ & $28(3)$ & $62(4)$ & $-6(3)$ & $-13(3)$ & $11(3)$ \\
\hline$C(104)$ & $38(3)$ & $46(3)$ & $59(4)$ & $-7(3)$ & $5(3)$ & $13(3)$ \\
\hline$C(105)$ & $38(3)$ & $37(3)$ & $38(3)$ & $1(2)$ & $0(2)$ & $2(2)$ \\
\hline$C(106)$ & $78(5)$ & $77(6)$ & $78(5)$ & $14(4)$ & $-1(4)$ & $-16(4)$ \\
\hline$C(107)$ & $108(7)$ & $91(6)$ & $66(5)$ & $-15(5)$ & $1(5)$ & $-16(5)$ \\
\hline$C(108)$ & $97(6)$ & $92(6)$ & $52(4)$ & $-8(4)$ & 2(4) & $-32(5)$ \\
\hline C(109) & $89(6)$ & $109(7)$ & $50(4)$ & $26(4)$ & $-21(4)$ & $-48(5)$ \\
\hline$C(110)$ & $79(5)$ & $102(6)$ & $62(5)$ & $30(4)$ & $-5(4)$ & $8(5)$ \\
\hline$C(111)$ & $54(4)$ & $143(8)$ & $58(4)$ & $35(5)$ & $5(3)$ & $22(5)$ \\
\hline$C(112)$ & $119(8)$ & $232(13)$ & $110(7)$ & $55(8)$ & $49(6)$ & $96(8)$ \\
\hline$C(113)$ & $50(4)$ & $65(4)$ & $92(5)$ & $-41(4)$ & $41(4)$ & $-20(4)$ \\
\hline$C(114)$ & $43(4)$ & $126(8)$ & $67(5)$ & $-6(5)$ & $18(3)$ & $13(5)$ \\
\hline$C(115)$ & $44(4)$ & $105(7)$ & $86(6)$ & $29(5)$ & $9(4)$ & $4(4)$ \\
\hline$C(116)$ & $65(4)$ & $49(4)$ & $97(5)$ & $13(4)$ & $12(4)$ & $-8(3)$ \\
\hline$C(117)$ & $61(4)$ & $42(4)$ & $60(4)$ & $-1(3)$ & $9(3)$ & $-9(3)$ \\
\hline$C(118)$ & $59(4)$ & $46(4)$ & 61(4) & $13(3)$ & $38(3)$ & $12(3)$ \\
\hline$C(119)$ & $100(5)$ & $77(5)$ & $81(5)$ & $23(4)$ & $59(4)$ & $31(4)$ \\
\hline
\end{tabular}


Table 5. Hydrogen coordinates ( $\left.\times 10^{4}\right)$ and isotropic displacement parameters $\left(\AA^{2} \times 10^{3}\right)$ for $[(\mathrm{BINAP}) \mathrm{Pd}]_{2}(\mathrm{dba})$.

\begin{tabular}{|c|c|c|c|c|}
\hline & $\mathrm{x}$ & $\mathrm{y}$ & z & $\mathrm{U}(\mathrm{eq})$ \\
\hline $\mathrm{H}(2 \mathrm{~A})$ & 11267 & 5345 & 7837 & 41 \\
\hline $\mathrm{H}(3 \mathrm{~A})$ & 8869 & 5459 & 7354 & 53 \\
\hline $\mathrm{H}(4 \mathrm{~A})$ & 7887 & 6334 & 7198 & 60 \\
\hline $\mathrm{H}(5 \mathrm{~A})$ & 9286 & 7120 & 7548 & 60 \\
\hline $\mathrm{H}(6 \mathrm{~A})$ & 11653 & 7019 & 8074 & 43 \\
\hline $\mathrm{H}(8 \mathrm{~A})$ & 15485 & 6552 & 7695 & 39 \\
\hline $\mathrm{H}(9 \mathrm{~A})$ & 16429 & 7451 & 7666 & 61 \\
\hline $\mathrm{H}(10 \mathrm{~A})$ & 15956 & 8138 & 8374 & 65 \\
\hline $\mathrm{H}(11 \mathrm{~A})$ & 14487 & 7956 & 9103 & 58 \\
\hline $\mathrm{H}(12 \mathrm{~A})$ & 13538 & 7069 & 9136 & 40 \\
\hline $\mathrm{H}(16 \mathrm{~A})$ & 16904 & 5742 & 10841 & 34 \\
\hline $\mathrm{H}(17 \mathrm{~A})$ & 16817 & 5395 & 11872 & 40 \\
\hline $\mathrm{H}(18 \mathrm{~A})$ & 14667 & 5069 & 12089 & 45 \\
\hline $\mathrm{H}(19 \mathrm{~A})$ & 12635 & 5113 & 11273 & 41 \\
\hline $\mathrm{H}(21 \mathrm{~A})$ & 11396 & 5379 & 10135 & 33 \\
\hline $\mathrm{H}(22 \mathrm{~A})$ & 11451 & 5719 & 9095 & 31 \\
\hline $\mathrm{H}(26 \mathrm{~A})$ & 15295 & 6766 & 10394 & 34 \\
\hline $\mathrm{H}(27 \mathrm{~A})$ & 16282 & 7597 & 10820 & 44 \\
\hline $\mathrm{H}(28 \mathrm{~A})$ & 18393 & 7913 & 10576 & 49 \\
\hline $\mathrm{H}(29 \mathrm{~A})$ & 19477 & 7406 & 9885 & 47 \\
\hline $\mathrm{H}(31 \mathrm{~A})$ & 19575 & 6554 & 9209 & 42 \\
\hline $\mathrm{H}(32 \mathrm{~A})$ & 18700 & 5698 & 8817 & 39 \\
\hline $\mathrm{H}(34 \mathrm{~A})$ & 13863 & 4374 & 8632 & 50 \\
\hline $\mathrm{H}(35 \mathrm{~A})$ & 12931 & 3748 & 9281 & 72 \\
\hline $\mathrm{H}(36 \mathrm{~A})$ & 13979 & 3632 & 10385 & 68 \\
\hline $\mathrm{H}(37 \mathrm{~A})$ & 16030 & 4119 & 10858 & 59 \\
\hline $\mathrm{H}(38 \mathrm{~A})$ & 17051 & 4720 & 10201 & 39 \\
\hline $\mathrm{H}(40 \mathrm{~A})$ & 18906 & 4702 & 9631 & 72 \\
\hline $\mathrm{H}(41 \mathrm{~A})$ & 20838 & 4207 & 9463 & 84 \\
\hline $\mathrm{H}(42 \mathrm{~A})$ & 20889 & 3874 & 8413 & 73 \\
\hline
\end{tabular}




\begin{tabular}{|c|c|c|c|}
\hline $\mathrm{H}(43 \mathrm{~A})$ & 19094 & 4122 & 7517 \\
\hline $\mathrm{H}(44 \mathrm{~A})$ & 17135 & 4603 & 7704 \\
\hline $\mathrm{H}(46 \mathrm{~A})$ & 11133 & 4415 & 4131 \\
\hline $\mathrm{H}(47 \mathrm{~A})$ & 12329 & 3573 & 4146 \\
\hline $\mathrm{H}(48 \mathrm{~A})$ & 14504 & 3449 & 4865 \\
\hline $\mathrm{H}(49 \mathrm{~A})$ & 15571 & 4189 & 5509 \\
\hline $\mathrm{H}(50 \mathrm{~A})$ & 14407 & 5039 & 5501 \\
\hline $\mathrm{H}(52 \mathrm{~A})$ & 10529 & 4388 & 5265 \\
\hline $\mathrm{H}(53 \mathrm{~A})$ & 8746 & 4158 & 5817 \\
\hline $\mathrm{H}(54 \mathrm{~A})$ & 7403 & 4854 & 6160 \\
\hline $\mathrm{H}(55 \mathrm{~A})$ & 7853 & 5781 & 5970 \\
\hline $\mathrm{H}(56 \mathrm{~A})$ & 9686 & 6026 & 5459 \\
\hline $\mathrm{H}(60 \mathrm{~A})$ & 12481 & 5796 & 2394 \\
\hline $\mathrm{H}(61 \mathrm{~A})$ & 11307 & 6135 & 1383 \\
\hline $\mathrm{H}(62 \mathrm{~A})$ & 9024 & 6502 & 1234 \\
\hline $\mathrm{H}(63 \mathrm{~A})$ & 7863 & 6472 & 2106 \\
\hline $\mathrm{H}(65 \mathrm{~A})$ & 7802 & 6177 & 3249 \\
\hline $\mathrm{H}(66 \mathrm{~A})$ & 8881 & 5784 & 4246 \\
\hline $\mathrm{H}(70 \mathrm{~A})$ & 11506 & 4786 & 2851 \\
\hline $\mathrm{H}(71 \mathrm{~A})$ & 12157 & 3941 & 2462 \\
\hline $\mathrm{H}(72 \mathrm{~A})$ & 14522 & 3641 & 2733 \\
\hline $\mathrm{H}(73 \mathrm{~A})$ & 16198 & 4183 & 3396 \\
\hline $\mathrm{H}(75 \mathrm{~A})$ & 16880 & 5068 & 4009 \\
\hline $\mathrm{H}(76 \mathrm{~A})$ & 16298 & 5919 & 4384 \\
\hline $\mathrm{H}(78 \mathrm{~A})$ & 13524 & 6797 & 3042 \\
\hline $\mathrm{H}(79 \mathrm{~A})$ & 12080 & 7394 & 2300 \\
\hline $\mathrm{H}(80 \mathrm{~A})$ & 10330 & 7895 & 2643 \\
\hline $\mathrm{H}(81 \mathrm{~A})$ & 10052 & 7825 & 3738 \\
\hline $\mathrm{H}(82 \mathrm{~A})$ & 11471 & 7222 & 4476 \\
\hline $\mathrm{H}(84 \mathrm{~A})$ & 15290 & 7342 & 3825 \\
\hline $\mathrm{H}(85 \mathrm{~A})$ & 17510 & 7768 & 4147 \\
\hline $\mathrm{H}(86 \mathrm{~A})$ & 19011 & 7543 & 5144 \\
\hline $\mathrm{H}(87 \mathrm{~A})$ & 18273 & 6910 & 5853 \\
\hline $\mathrm{H}(88 \mathrm{~A})$ & 16065 & 6500 & 5565 \\
\hline $\mathrm{H}(89 \mathrm{~A})$ & 15368 & 4883 & 6780 \\
\hline $\mathrm{H}(90 \mathrm{~A})$ & 12583 & 5274 & 6694 \\
\hline
\end{tabular}




\begin{tabular}{|c|c|c|c|c|}
\hline $\mathrm{H}(92 \mathrm{~A})$ & 12317 & 6322 & 6606 & 31 \\
\hline $\mathrm{H}(93 \mathrm{~A})$ & 14667 & 6878 & 6288 & 29 \\
\hline $\mathrm{H}(95 \mathrm{~A})$ & 15757 & 3889 & 6929 & 47 \\
\hline $\mathrm{H}(96 \mathrm{~A})$ & 14988 & 2982 & 7067 & 65 \\
\hline $\mathrm{H}(97 \mathrm{~A})$ & 12732 & 2828 & 7221 & 66 \\
\hline $\mathrm{H}(98 \mathrm{~A})$ & 11212 & 3583 & 7237 & 65 \\
\hline H(99A) & 11976 & 4488 & 7114 & 48 \\
\hline $\mathrm{H}(10 \mathrm{~B})$ & 14121 & 7762 & 5607 & 50 \\
\hline $\mathrm{H}(10 \mathrm{C})$ & 12898 & 8592 & 5443 & 64 \\
\hline $\mathrm{H}(10 \mathrm{D})$ & 10844 & 8708 & 5846 & 62 \\
\hline $\mathrm{H}(10 \mathrm{E})$ & 10060 & 7976 & 6420 & 58 \\
\hline $\mathrm{H}(10 \mathrm{~F})$ & 11273 & 7133 & 6562 & 47 \\
\hline $\mathrm{H}(10 \mathrm{G})$ & 9074 & 3099 & 2073 & 96 \\
\hline $\mathrm{H}(10 \mathrm{H})$ & 10563 & 2833 & 1408 & 109 \\
\hline $\mathrm{H}(10 \mathrm{I})$ & 11292 & 3491 & 727 & 99 \\
\hline $\mathrm{H}(10 \mathrm{~J})$ & 10691 & 4421 & 795 & 106 \\
\hline $\mathrm{H}(11 \mathrm{~B})$ & 9235 & 4701 & 1510 & 101 \\
\hline $\mathrm{H}(11 \mathrm{C})$ & 7824 & 3759 & 2556 & 225 \\
\hline $\mathrm{H}(11 \mathrm{D})$ & 7234 & 4268 & 2071 & 225 \\
\hline $\mathrm{H}(11 \mathrm{E})$ & 8605 & 4352 & 2654 & 225 \\
\hline $\mathrm{H}(11 \mathrm{~F})$ & 2714 & 6681 & 10481 & 78 \\
\hline $\mathrm{H}(11 \mathrm{G})$ & 1669 & 7301 & 9669 & 93 \\
\hline $\mathrm{H}(11 \mathrm{H})$ & 1877 & 8249 & 9840 & 95 \\
\hline $\mathrm{H}(11 \mathrm{I})$ & 3099 & 8583 & 10862 & 86 \\
\hline $\mathrm{H}(11 \mathrm{~J})$ & 4245 & 7976 & 11675 & 66 \\
\hline $\mathrm{H}(11 \mathrm{~K})$ & 4235 & 6472 & 11546 & 120 \\
\hline $\mathrm{H}(11 \mathrm{~L})$ & 4065 & 6908 & 12111 & 120 \\
\hline $\mathrm{H}(11 \mathrm{M})$ & 5404 & 6945 & 11778 & 120 \\
\hline
\end{tabular}




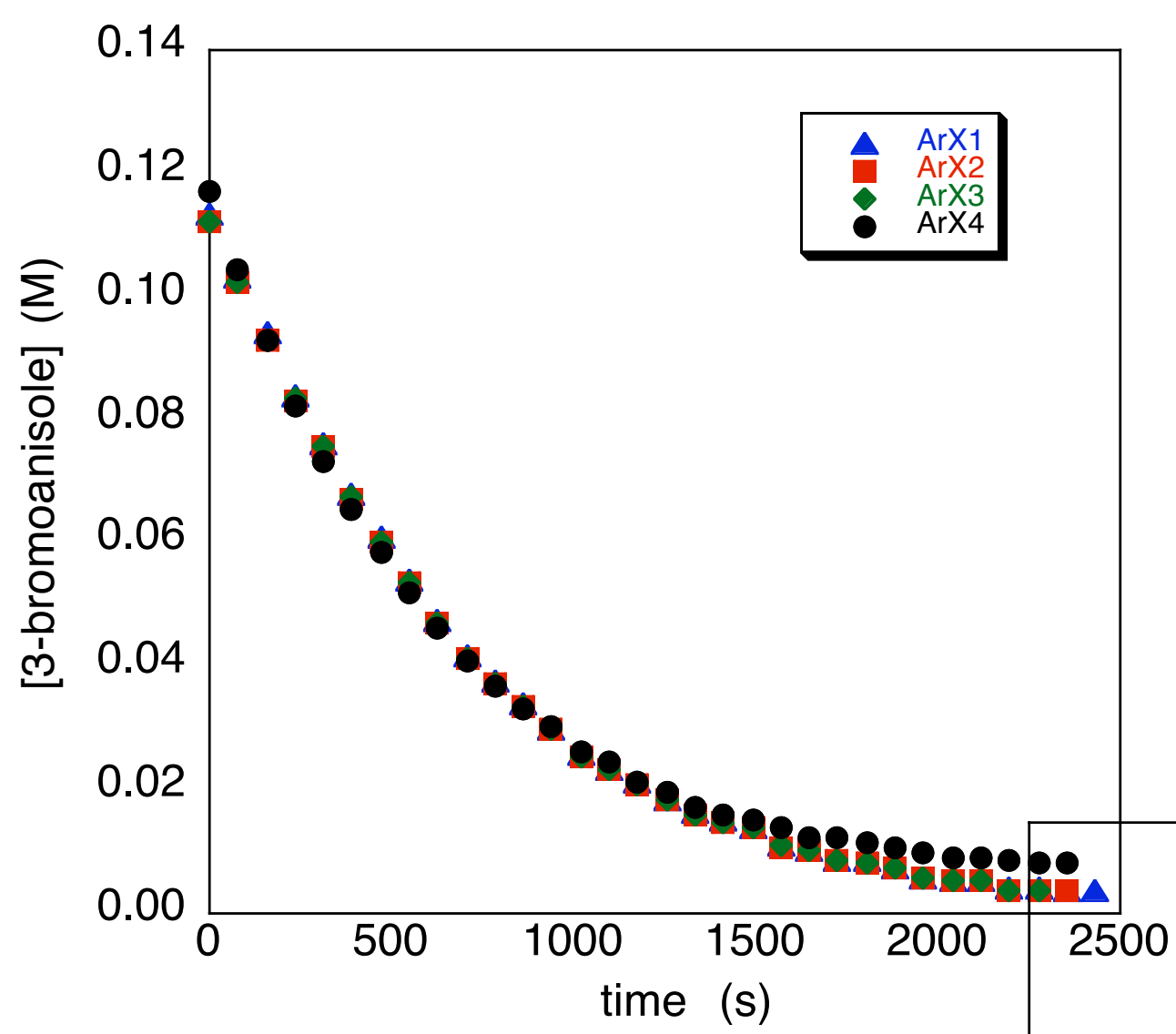

Figure S1. Sequential reactions of 3-bromoanisole $(0.12 \mathrm{M})$ with $N$-methylpiperazine $(0.93 \mathrm{M})$ and NaOtAm 1.0 $\mathrm{M}$ as base with Pd(BINAP $)_{2}(10 \mathrm{mM})$ and BINAP $(2.0 \mathrm{mM})$ as catalyst at $70^{\circ} \mathrm{C}$. ArX1, ArX2, ArX3 and ArX4 correspond to the decay of the $1^{\text {st }}, 2^{\text {nd }}$, $3^{\text {rd }}$ and $4^{\text {th }}$ portions of 3 -bromoanisole. 


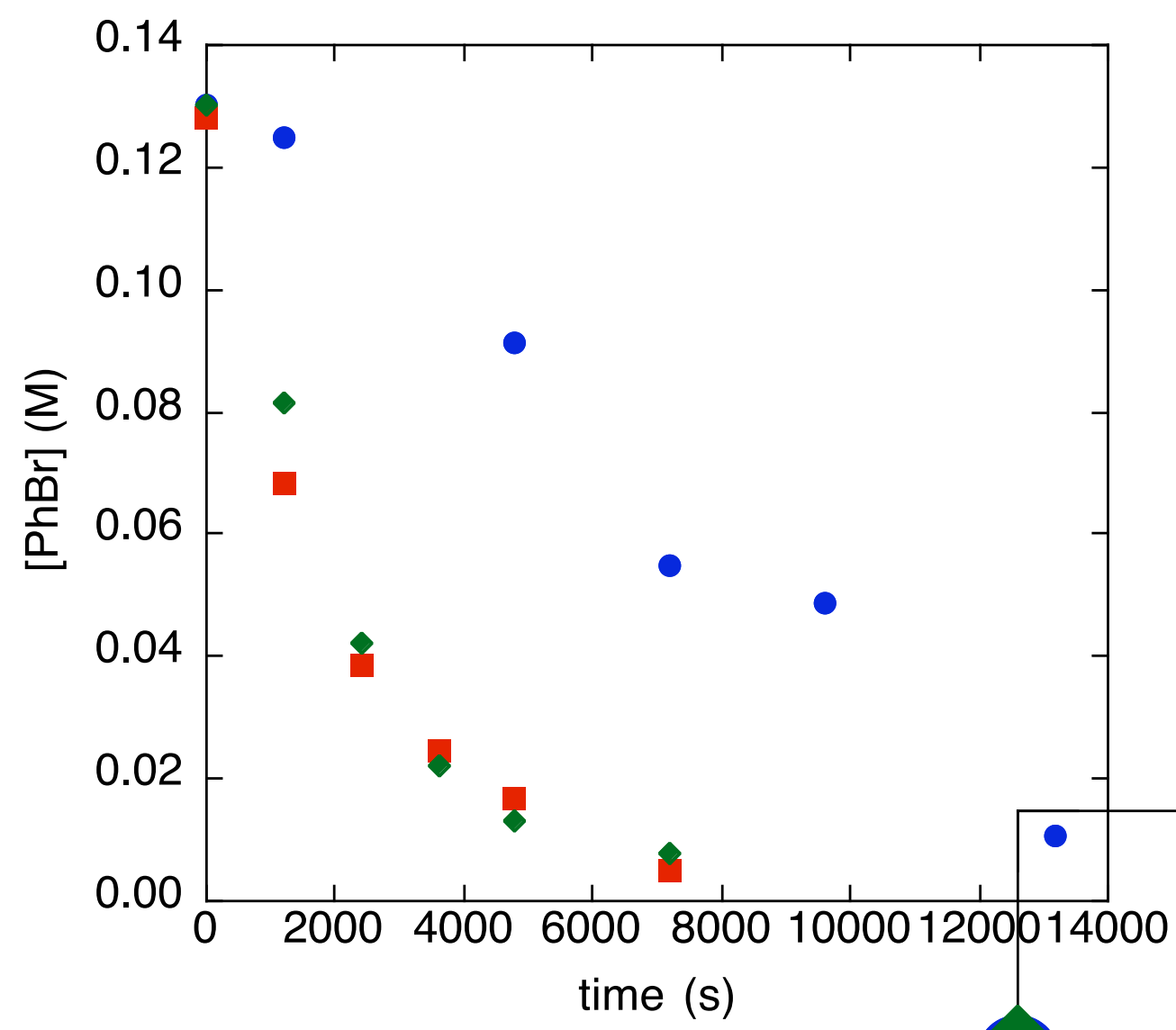

Figure S2. Sequential reactions of bromobenzene $(0.13 \mathrm{M})$ with hexylamine $(0.93 \mathrm{M})$ and NaOtAm $1.0 \mathrm{M}$ as base with $\mathrm{Pd}_{2}(\mathrm{dba})_{3}(5 \mathrm{mM})$ and BINAP $(10 \mathrm{mM})$ as catalyst at $60^{\circ} \mathrm{C}$. ArX1, ArX2 and ArX3 correspond to the decay of the $1^{\text {st }}, 2^{\text {nd }}$ and $3^{\text {rd }}$ portions of bromobenzene. 


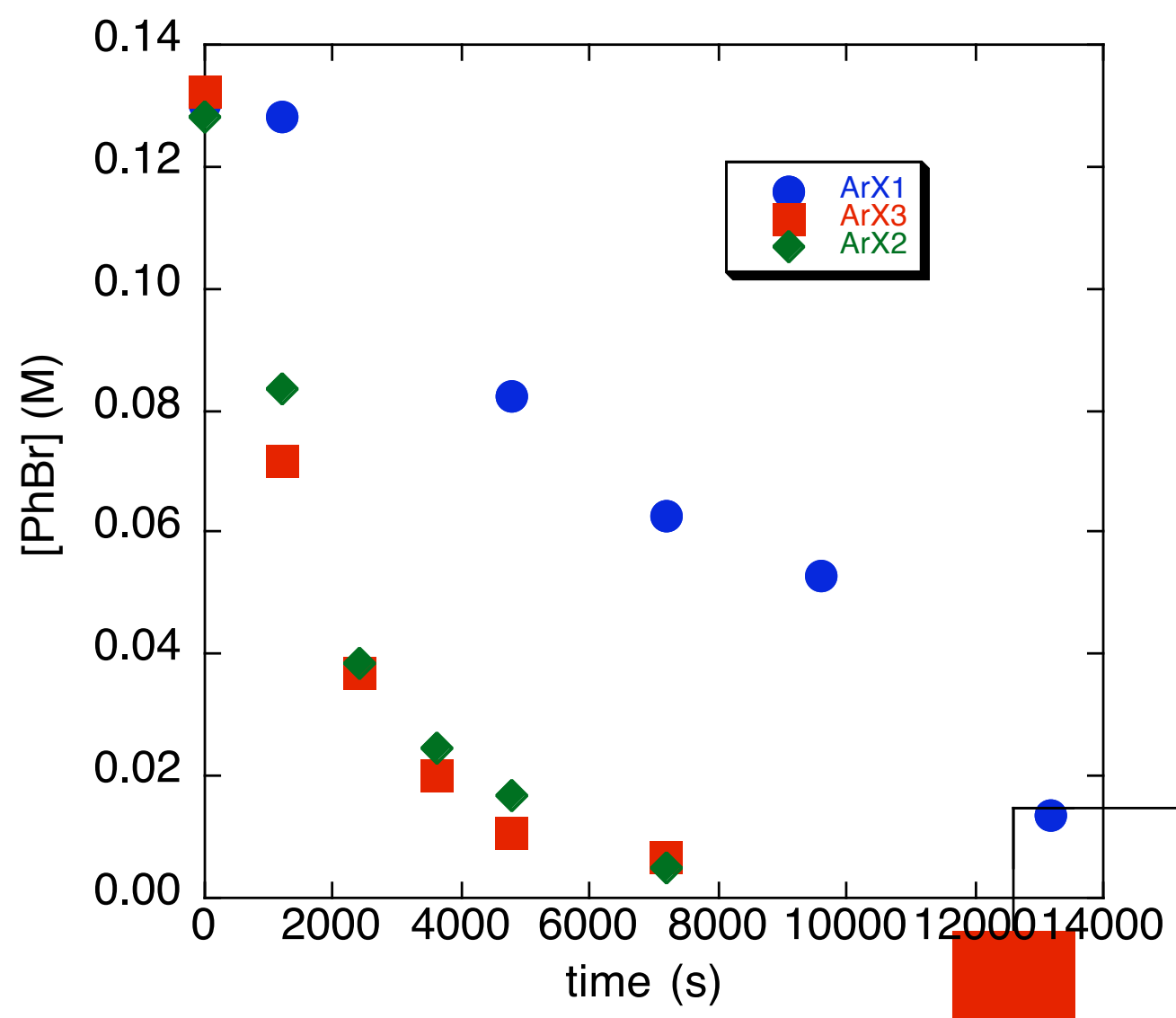

Figure S3. Sequential reactions of bromobenzene $(0.13 \mathrm{M})$ with $N$-methylpiperazine $(0.93 \mathrm{M})$ and $\mathrm{NaOtAm} 1.0 \mathrm{M}$ as base with $\operatorname{Pd}_{2}(\mathrm{dba})_{3}(5 \mathrm{mM})$ and BINAP $(10 \mathrm{mM})$ as catalyst at $60^{\circ} \mathrm{C}$. ArX $1, \operatorname{ArX} 2$ and ArX3 correspond to the decay of the $1^{\text {st }}, 2^{\text {nd }}$ and $3^{\text {rd }}$ portions of bromobenzene. 


\section{Derivation of rate equations.}

The rate equation corresponds to the pathway in which $\mathrm{PdL}_{2}$ lies off of the catalytic cycle, as shown in Scheme 4.

Steady state kinetics were used. These conditions are valid because no induction period is observed when $2 \mathrm{mM}$ BINAP is present.

$\frac{-\mathrm{d}[\mathrm{ArX}]}{\mathrm{dt}}=\frac{\mathrm{k}_{1} \mathrm{k}_{2} \mathrm{k}_{3} \mathrm{k}_{4}[\mathrm{ArBr}][\text { amine }][\text { base }][\mathrm{Pd}]}{\mathrm{k}_{-1} \mathrm{k}_{3} \mathrm{k}_{4}[\text { amine }][\text { base }][\mathrm{L}]+\mathrm{k}_{1} \mathrm{k}_{3} \mathrm{k}_{4}[\text { amine }][\text { base }]+\mathrm{k}_{1} \mathrm{k}_{2} \mathrm{k}_{4}[\mathrm{ArBr}]+\mathrm{k}_{1} \mathrm{k}_{2} \mathrm{k}_{3}[\mathrm{ArBr}][\text { amine }][\text { base }]}$

Approximations:

$\mathrm{k}_{-1} \mathrm{k}_{3} \mathrm{k}_{4}$ [amine][base][L] $>\mathrm{k}_{1} \mathrm{k}_{2} \mathrm{k}_{4}[\mathrm{ArBr}]$ because $\mathrm{k}_{2}[\mathrm{ArBr}] \sim \mathrm{k}_{-1}[\mathrm{~L}]$ and $\mathrm{k}_{3}$ [amine] [base $>>\mathrm{k}_{1}$ The reaction of amine and base with $[\mathrm{Pd}(\mathrm{BINAP})(\mathrm{Ar})(\mathrm{Br})]$ occurs rapidly at room temperature, but dissociation of BINAP requires over the time frame of hours at $45^{\circ} \mathrm{C}$.

$k_{-1} k_{3} k_{4}\left[\right.$ amine][base][L] $\gg k_{1} k_{3} k_{4}$ [amine][base] because $k_{-1}[L] \gg k_{1}$ The equilibrium for dissociation of BINAP is known to be very small $\left(k_{-1}>>k_{1}\right)$ because no accumulation of free BINAP and $[\mathrm{Pd}(\mathrm{BINAP})]$ is observed in the ${ }^{1} \mathrm{H}$ or ${ }^{31} \mathrm{P}$ NMR spectra of $\left[\mathrm{Pd}(\mathrm{BINAP})_{2}\right]$

$\mathrm{k}_{-1} \mathrm{k}_{3} \mathrm{k}_{4}\left[\right.$ amine][base][L] $\gg \mathrm{k}_{1} \mathrm{k}_{2} \mathrm{k}_{3}[\mathrm{ArBr}]\left[\right.$ amine][base] because $\mathrm{k}_{2}[\mathrm{ArBr}] \sim \mathrm{k}_{-1}[\mathrm{~L}]$ and $\mathrm{k}_{4} \gg \mathrm{k}_{1}$ The reductive elimination of $N$-alkyl arylamines from BINAP complexes occurs rapidly at room temperature (the reaction of amine and base with [Pd(BINAP)(Ar)(Br)] occurs rapidly at room temperature to form the arylamine product), while the dissociation of BINAP occurs on the timescale of hours at $45^{\circ} \mathrm{C}$.

With these approximations, equation (1) can be reduced to

$\frac{-\mathrm{d}[\mathrm{ArX}]}{\mathrm{dt}}=\frac{\mathrm{k}_{1} \mathrm{k}_{2}[\mathrm{ArBr}][\mathrm{Pd}]}{\mathrm{k}_{-1}[\mathrm{~L}]}$

Integrating both sides,

$[\operatorname{ArX}]=[\operatorname{ArX}]_{0} \exp \left\{\left(\frac{-\mathrm{k}_{1}}{[\mathrm{~L}]}\right)\left(\frac{\mathrm{k}_{2}}{\mathrm{k}_{-1}}\right)[\mathrm{Pd}] \mathrm{t}\right\}$

From data on the stoichiometric oxidative addition of 3-bromoanisole to $\left[\mathrm{Pd}(\mathrm{BINAP})_{2}\right]$ at $70{ }^{\circ} \mathrm{C}$ shown in Figure $\mathrm{S} 4, \mathrm{k}_{2} / \mathrm{k}_{-1}=0.042$ and $\mathrm{k}_{1}=4.9 \times 10^{-3} \mathrm{~s}^{-1}$. With these values of rate constants and the integrated equation predicted values of $t$ from the values of $[\mathrm{ArBr}]$ were used to generate a plot of $[\mathrm{ArX}]$ vs $\mathrm{t}$.

$\mathrm{PdL}_{2}$-On Model (Scheme 3) 
$\frac{-d[A r X]}{d t}=\frac{k_{1}\left(\frac{k_{2}}{k_{-1}}\right)[A r B r][P d]}{[L]+\left(\frac{k_{2}}{k_{-1}}\right)[\operatorname{ArBr}]}$

Integrating both sides,

$$
\left\{\frac{[\mathrm{L}]}{\mathrm{k}_{1}\left(\frac{\mathrm{k}_{2}}{\mathrm{k}_{-1}}\right)[\mathrm{Pd}]}\right\} \ln \left(\frac{[\mathrm{ArX}]_{\mathrm{o}}}{[\mathrm{ArX}]}\right)+\frac{1}{\mathrm{k}_{1}[\mathrm{Pd}]}\left([\mathrm{ArX}]_{\mathrm{O}}-[\mathrm{ArX}]\right)=\mathrm{t}
$$

This equation was used with the rate constants from the plot in Figure S4 and known values of $[\mathrm{ArX}]$ to calculate predicted values of $\mathrm{t}$ to generate a plot of $[\mathrm{ArX}]$ vs $\mathrm{t}$. 